\title{
Terahertz-Wave-Plethysmography (TPG): A New Principle of Radar Based Pulse Detection
}

\section{Yu Rong ( $\nabla$ yrong5@asu.edu )}

Arizona State University

Panagiotis C. Theofanopoulos

Arizona State University

Georgios C. Trichopoulos

Arizona State University

Daniel W. Bliss

Arizona State University

\section{Research Article}

Keywords: Terahertz-Wave-Plethysmography (TPG), Radar Based Pulse Detection, frequency band, noncontact cardiac sensing application

Posted Date: December 16th, 2021

DOI: https://doi.org/10.21203/rs.3.rs-1109431/v1

License: (c) (1) This work is licensed under a Creative Commons Attribution 4.0 International License.

Read Full License 


\title{
Terahertz-Wave-Plethysmography (TPG): A New Principle of Radar Based Pulse Detection
}

\author{
Yu Rong ${ }^{1,+}$, Panagiotis C. Theofanopoulos ${ }^{2,+}$, Georgios C. Trichopoulos ${ }^{2,+}$, and Daniel W. \\ Bliss $^{1,+}$
}

${ }^{1}$ Center for Wireless Information Systems and Computational Architectures (WISCA) at the School of Electrical, Computer and Energy Engineering, Arizona State University, Tempe, AZ 85281 USA

${ }^{2}$ Terahertz Research Lab at the School of Electrical, Computer and Energy Engineering, Arizona State University, Tempe, AZ 85281 USA

*corresponding yrong5@asu.edu

\begin{abstract}
This study presents findings at Terahertz (THz) frequency band for non-contact cardiac sensing application. For the first time, cardiac pulse information is simultaneously extracted using $\mathrm{THz}$ waves based on the two established principles in electronics and optics. The first fundamental principle is micro-Doppler $(\mathrm{mD})$ motion effect, initially introduced in coherent laser radar system $^{1,2}$ and first experimentally demonstrated for vital sign detection ${ }^{3}$. This motion based method, primarily using coherent phase information from the radar receiver, has been widely exploited in microwave frequency bands and has recently found popularity in millimeter waves (mmWave). The second fundamental principle is reflectance based optical measurement using infrared or visible light. The variation in the light reflection is proportional to the volumetric change of the heart, often referred as photoplethysmography (PPG). PPG has been a popular technology for pulse diagnosis. Recently it has been widely incorporated into various smart wearables for long-term monitoring, such fitness training and sleep monitoring. Herein, the concept of Terahertz-Wave-Plethysmography (TPG) is introduced, which detects blood volume changes in the upper dermis tissue layer by measuring the reflectance of THz waves, similar to the existing remote PPG (rPPG) principle ${ }^{4}$. The TPG principle is justified by scientific deduction, electromagnetic wave (EM) simulations and carefully designed experimental demonstrations. Additionally, pulse measurements from various peripheral body parts of interest (BOI), palm, inner elbow, temple, fingertip and forehead, are demonstrated using a wideband THz sensing system developed by Terahertz Electronics Lab at Arizona State University (ASU), Tempe. Among the BOls under test, it is found that the measurements from forehead BOI gives the best accuracy with mean heart rate (HR) estimation error 1.51 beats per minute (BPM) and stand deviation (std) 1.08 BPM. The results validate the feasibility of radar based plethysmography for direct pulse monitoring. Finally, a comparative study on pulse sensitivity in TPG and rPPG is conducted. The results indicate that the TPG contains more pulsatile from the forehead BOI than that in the rPPG signals and thus generate better heart rate (HR) estimation statistic in the form of empirical cumulative distribution function (CDF) of HR estimation error.
\end{abstract}

\section{Introduction}

Microwave (1 GHz to $60 \mathrm{GHz}$ ) radars are widely used for detection of human vital signs, such as heart rate (HR), breathe rate (BR) and body temperature ${ }^{5,6}$, which are important biometrics for healthcare development ${ }^{7-9}$. Explicitly, these systems leverage advanced signal processing techniques such as complex signal demodulation ${ }^{10}$ and phase-based methods ${ }^{11-14}$ to extract vital signs from the captured backscattered signals. These methods were initially developed for narrowband systems and later extended to wideband radars offering better clutter performance. However, the robustness of these systems is hindered by certain limitations arising due to low operation frequency and limited radio frequency (RF) resources in these bands. The developed Doppler signal processing techniques have difficultly providing accurate pulse measurements when dynamic breathing pattern is present ${ }^{15}$, not to mention the presence of other sources of random body motion artifacts ${ }^{16-18}$. The fractional bandwidth (BW) of these frequencies leads to clutter noise due to low resolution range bins, especially in crowded environments/targets. For example, a $15 \% \mathrm{BW}$ at $60 \mathrm{GHz}$ is $9 \mathrm{GHz}$, leading to 1.7 centimeters $(\mathrm{cm})$ range resolution. However, the heart motions on the body surface are less than 1 millimeter $(\mathrm{mm})$, hence the signal of the cardiac pulses is hard to detect within such wide range bins that include breathing motions (a few $\mathrm{mm}$ to $1 \mathrm{~cm}^{19}$ ) and other body motions, along with clutter noise from other scatterers (e.g. clothing). In addition, the small apertures needed for mobile applications or embedded systems, lead to wide beams that capture the backscattered signals of multiple targets, further increasing clutter noise. Even though these issues are addressed exploring active motion cancellation and sensor fusion techniques $10,17,18,20,21$, still these configurations are limited in complex target scenes where multiple scatterers are located within in the field-of-view (FOV). 


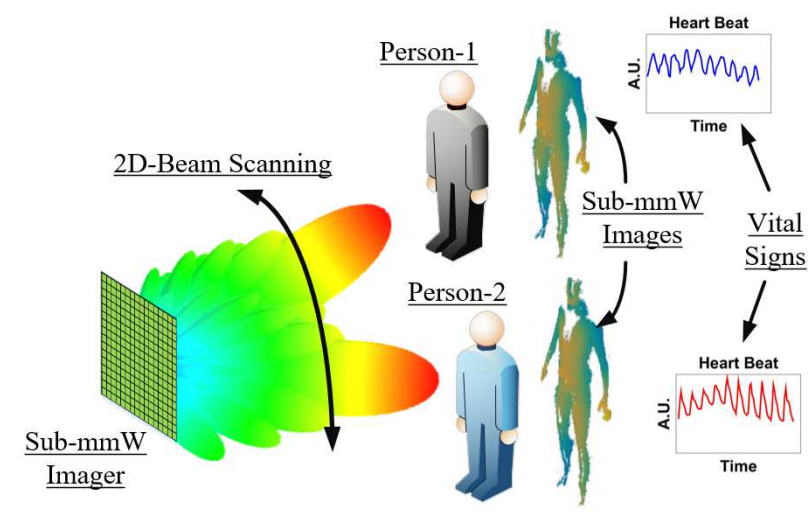

Figure 1. Exploiting highly directive 3D radar $\mathrm{THz}$ radars systems for enhanced imaging and vital sign extraction in cluttered environments.

Alternatively, the use of higher-operating frequencies such as mmWave and $\mathrm{THz}(100 \mathrm{GHz}-10 \mathrm{THz})$ could potentially alleviate the aforementioned limitations. Namely, these non-ionizing frequencies offer large bandwidth that allow for increased range resolution, thus less cluttering noise. For instance, a $15 \%$ fractional $\mathrm{BW}$ at $300 \mathrm{GHz}$ is $45 \mathrm{GHz}$, resulting into a $3.3 \mathrm{~mm}$ range resolution. In addition, small physical apertures are electrically larger in these frequencies (compared to microwaves) due to the small wavelength, leading to narrow beams that can be focused only on one target/person, thus further decreasing cluttering noise from undesired scatterers. Furthermore, the small motions caused by the pulse related surface skin motion and blood flow on/near the body surface, can be easily detected with phase-based methods, since the phase sensitivity increases due to the smaller wavelength. For example, a $0.5 \mathrm{~mm}$ motion causes a phase change of 3.6 degrees at $60 \mathrm{GHz}$, while the respective phase change at $300 \mathrm{GHz}$ is 18 degrees. This allows for the detection of heart caused micro-motions even in peripheral body sites based on $\mathrm{mD}$ motion effect ${ }^{22}$. Breathing-interference-free pulse measurement is possible at these sites because they are further away from the upper torso body area.

In combination with the aforementioned, these higher-operating frequencies are also exploited for high spatial resolution 3D imaging ${ }^{23-26}$ (both range and 2D cross-range), due to the directive beams and the large BW. Furthermore, instead of using complex and bulky mmWave/THz radars, low profile and complexity imagers can be utilized ${ }^{27-29}$ (e.g. reflecarrays) to form scannable narrow beams, offering more versatile vital signs monitoring (VSM) solutions. These systems can be deployed and form 3D images of various targets, and then by analyzing the captured data, identify the vital signs of each person within FOV, as shown in Fig. 1. Moreover, at short distances these systems can be leveraged to focus the beam on different parts of the human body detecting pulse information from multiple body parts offering new opportunities, including blood circulation inspection and remote blood pressure measurements even through clothes. Further valuable information from the pulse signal in addition to simple heart rate can be extracted for non-contact cardiovascular health assessment, such as vascular aging, artery stiffness.

In this work, a wideband sub-THz system is developed that forms narrow beams focusing the waves on different parts of the body and extract the pulse signals using the TPG concept based on the reflectivity changes in the magnitude response, rather than the $\mathrm{mD}$ phase ${ }^{11,14,30}$. As such, the recorded backscattered signals are leveraged to extract the vital signs of the person, using both the traditional micro-Doppler method and the herein introduced TPG. The unique nature of the THz waves, which lie between the microwave and the optical frequency regions, constitute them a unique frequency band for remote vital sign sensing using different methods. Moreover, due to the nature of each technique (mD-motion based vs TPG-reflectance based detection), the micro motions and -close to skin- blood concentration of each body part contribute differently on each method's accuracy. These measurement differences from the two methods are demonstrated in great detail and used as a cross-validation at the forehead BOI. Subsequently, the THz waves are focused on the temple, inner elbow, palm and fingertip to investigate their respective TPG vital sign detection capabilities. Distortionless vital sign sensing is possible by strategically choosing the body parts.

\section{Remote Cardiac Sensing from Microwave to Visible Light}

The spectral location of THz waves implies their unique electromagnetic (EM) properties, as they occupy the part of the spectrum between microwave bands and the visible lights (Fig. 2), which inherently possess the optical-like features from the visible light, and also the valuable phase information as seen in microwave band. Physiological measurements use each 


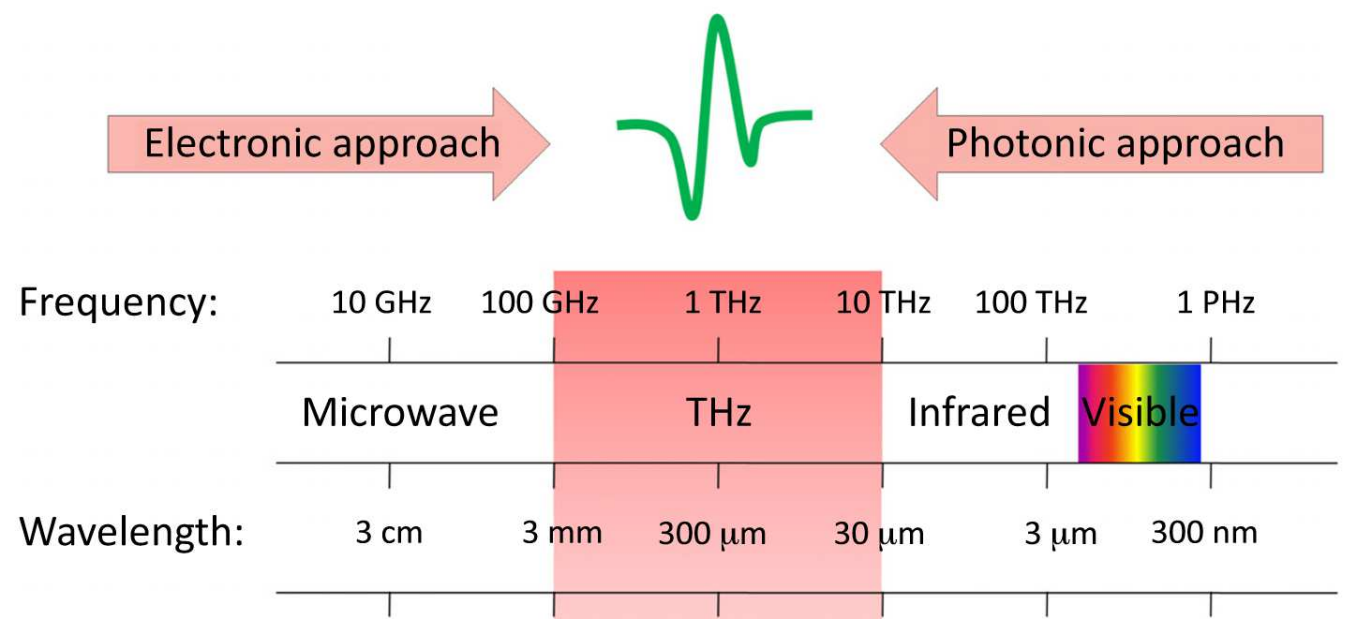

Figure 2. An example EM wave spectra of interest in this study.

of these frequency bands, ranging from microwave $\left(10^{9} \mathrm{~Hz}\right)^{3,15}$, mmWave $\left(10^{10} \mathrm{~Hz}\right)^{30,31}, \mathrm{THz}\left(10^{11} \mathrm{~Hz}\right)^{22,32-34}$, infrared $\left(10^{12} \mathrm{~Hz}\right)^{35,36}$ and visible light $\left(10^{14} \mathrm{~Hz}\right)^{37,38}$, have been reported previously.

Non-contact VSM technologies revolutionize the diagnosis experience among the patients and the medical professionals. It offers convenient and comfortable way to take HR and BR measurements over a long period of time since it does not require a direct physical contact. This could leads many useful health monitoring applications at residence house, such elderly care and infant monitoring. Various remote sensing devices are developed to demonstrate the possibility to detect cardiac pulse from a distance. The EM spectra of interest ranges from microwave a few Gigahz $\left(10^{9} \mathrm{~Hz}\right)$ to visible light about $1 \mathrm{Petahertz}\left(10^{15} \mathrm{~Hz}\right)$ and the corresponding wavelength is from a few $\mathrm{cms}$ to about 300 nanometers. These bands consist of non-ionizing radiations and thus can be safely used for biomedical applications. The significant results from the prior works ${ }^{3,15,30,32,34-40}$ in these spectral bands are reviewed, making this manuscript self-contained. After a comparative study, the main results of TPG will be introduced. This study focuses on understanding the physical principle rather than the signal processing techniques.

The EM spectra in Fig. 2 can be divided into three portions. The long-wavelength region (low frequency) covers microwave and mmWave; the short-wavelength region (high frequency) includes infrared and visible light. In between, THz radiation exhibits both photon-like and electron-like properties, which will be demonstrated in this study for pulse detection. Studies of the long-wavelength region rely predominantly on electronics, whereas studies of the short-wavelength region rely predominantly on photonics, resulting in a gap between these two research fields because of the limited availability of effective THz-generating sources and THz-sensitive detectors. But recently with the rapid development of $\mathrm{THz}$ science and technology, extensive research of biological effects of $\mathrm{THz}$ radiation has been conducted in the field of life science.

\section{Micro-Doppler Motion}

In the long-wavelength region, microwave and mmWave sensors were shown to detect human heartbeat based on the well-known $\mathrm{mD}$ principle. In 1975, a microwave technique was first proposed for measuring respiratory movements of humans and animals ${ }^{3}$. Later, an X-band life detection radar was developed for detecting heartbeat and breathing of human subjects ${ }^{41,42}$. More recently, mmWave multiple-input multiple-output (MIMO) sensors were shown to detect heartbeats from multiple subjects due to improved spatial degree of freedom and phase signal sensitivity ${ }^{30,31}$. An operational example of mD bio-radar is illustrated in Fig. 3. The transmit antenna sends a train of radar pulses towards the human subject. These pulses are reflected by the moving chest wall. The periodic expansion and contraction of the chest movement generates an observable change known as $\mathrm{mD}$ shift that is acquired by the receiving antenna. By processing the radar return, the vital sign signals are extracted. Often, phase information is used for vital sign detection because of the simple linear relation between the phase rotation and the skin/tissue/body motion from cardiovascular activity. The phase rotation depends on the operating frequency ${ }^{10,22}$.

$$
\phi=\frac{4 \pi x_{h}(t)}{\lambda}
$$

where $x_{h}(t)$ denotes the heartbeat motion and $\lambda$ denotes the wavelength. The major challenge for accurate HR detection using $\mathrm{mD}$ motion methods with microwave narrowband systems is motion coupling. The signal contains multitude stronger breathing signal and weak pulse signal of interest due to the aforementioned small BW and large FOV. In real situations, the precise pulse wave reconstruction is not possible except in some high signal-to-noise ratio region as in reference ${ }^{43}$. For long-term 


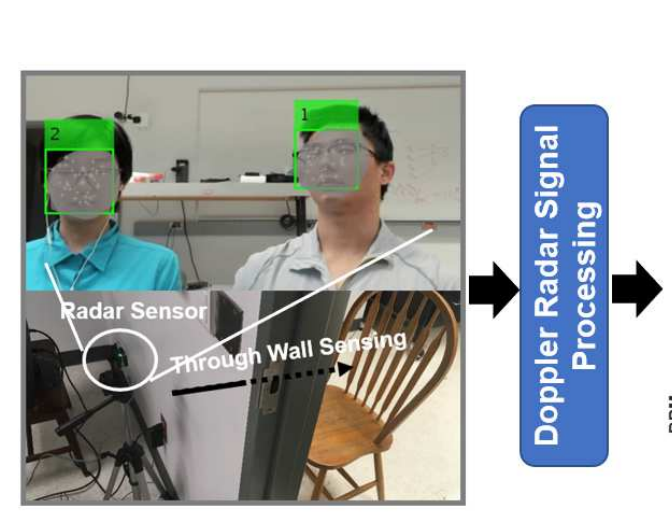

(a)
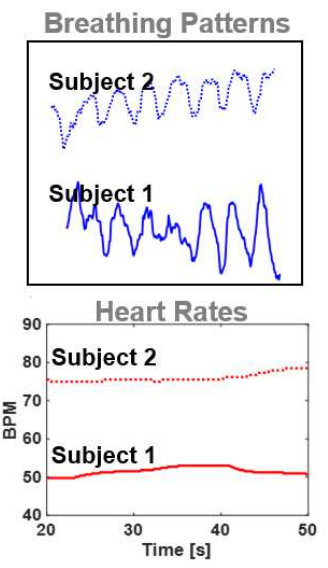

(n)

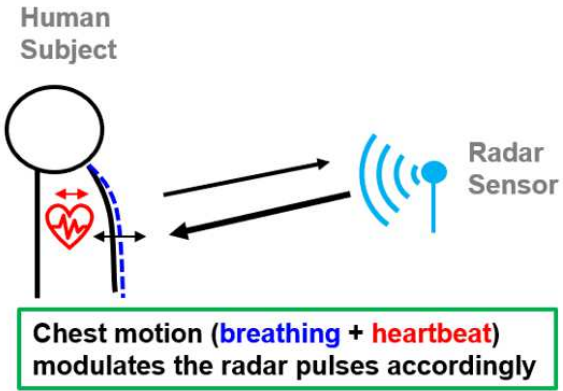

(b)

Figure 3. Working principle of example Doppler bio-radar system modified from reference ${ }^{55}$. (a) vital sign motions extraction using through-wall radar; (b) micro-Doppler of human vital signs measurement principle illustration.

monitoring application, random and involuntary body motion is inevitable. Significant efforts were devoted to dealing with motion interference ${ }^{44-46}$. Practical and robust methods for motion-tolerant pulse detection using long-wavelength signals remain an open question.

\section{Photoplethysmography}

In the short-wavelength region, photoplethysmography (PPG) is another non-invasive physical principle for monitoring pulse/HR. Similar to the gold standard for HR monitoring electrocardiography (ECG), PPG require direct physical contact with the subject. The popularity of the PPG technology as an alternative HR monitoring technique has recently increased, mainly due to the simplicity of its operation, the wearing comfortability for its users, and its cost effectiveness. The operation of reflectance-mode PPG requires two components, a light source and a photodetector. PPG relies on measuring the pulse-like variations in light absorption in an illuminated skin area caused by the difference in absorption curves of oxygenated and deoxygenated blood ${ }^{47}$. The amplitude of the variations depend on the amount of blood rushing into the peripheral vascular bed, the optical absorption of blood, skin pigmentation, ambient light and the wavelength used to illuminate the blood ${ }^{47-50}$. Different optical wavelengths (e.g. infrared, green) interact differently with blood and tissues, involving several physical processes, i.e. scattering, absorption, reflection, transmission and fluorescence ${ }^{51}$. In reflectance-mode, green light is one of the most commonly used colors because it contains more pulsatile information compared with other colors, according to the Fig. $4^{52}$. The reflectance PPG principle also motivated the use of digital cameras to measure plethysmographic signals from face or exposed skin videos under ambient light conditions $4,53,54$. This technique is often referred as remote PPG (rPPG) or imaging PPG. Recently infrared cameras is included in rPPG for heartbeat detection due to their resilience to low-light and variable-light conditions ${ }^{35,36}$. An example of rPPG using near-infrared camera is illustrated in Fig. 5.

\section{Comparison of VSM in EM Waves}

The EM spectra for VSM can be broadly divided into four categories, microwave (including mmWave), THz, infrared and visible light. Due to the aforementioned reasons, there is significantly less effort on VSM using THz. Before presenting the new insights on using THz for VSM, it is worthwhile to review the current advances of mD bio-radar sensors and rPPG optical sensors.

rPPG using optical sensors (infrared and visible light) are advantageous over microwave bio-radars for motion tolerance VSM. That is because rPPG signal is based on the changes in the skin reflectivity (or skin color shift) not from skin motion. High resolution images from optical sensors provide abundant information for signal processing. Other body motion artifacts can be separated via computer vision techniques by leveraging millions of image pixels, multiple available color channels from low-cost webcams. Infrared is heavily investigated due to privacy issue of using normal color cameras. In general, optical sensors do not penetrate many common materials, including clothes and blankets and are limited to line-of-sight (LOS) applications.

On the other hand, conventional narrowband $\mathrm{mD}$ radars with limited array size (mostly single antenna systems) are not able to handle realistic dynamic motion profile. Popular Doppler phase signal is more susceptible to chest motion and other random 


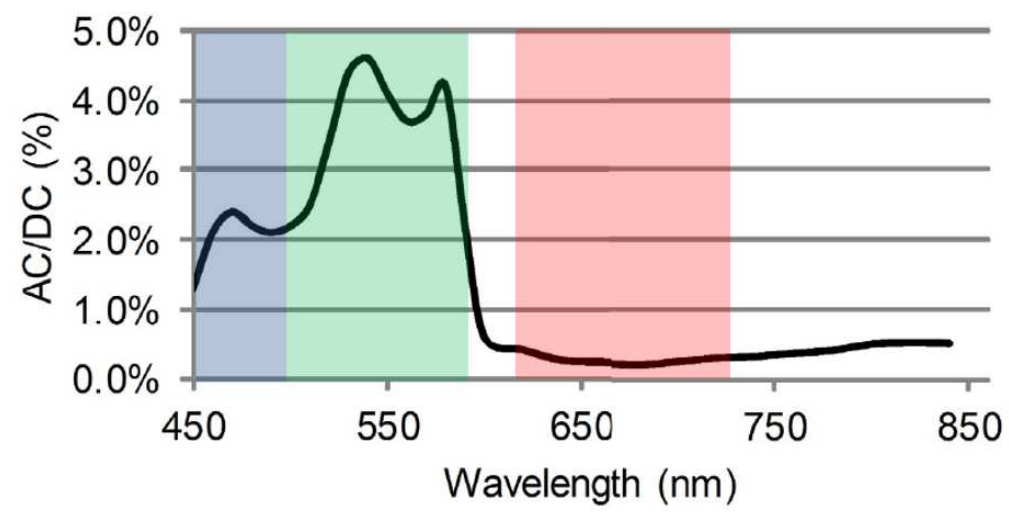

Figure 4. Wavelength dependence the AC/DC ratio of a reflectance contact PPG signal ${ }^{52}$. DC denotes the pulse baseline and AC the pulsatile amplitude.

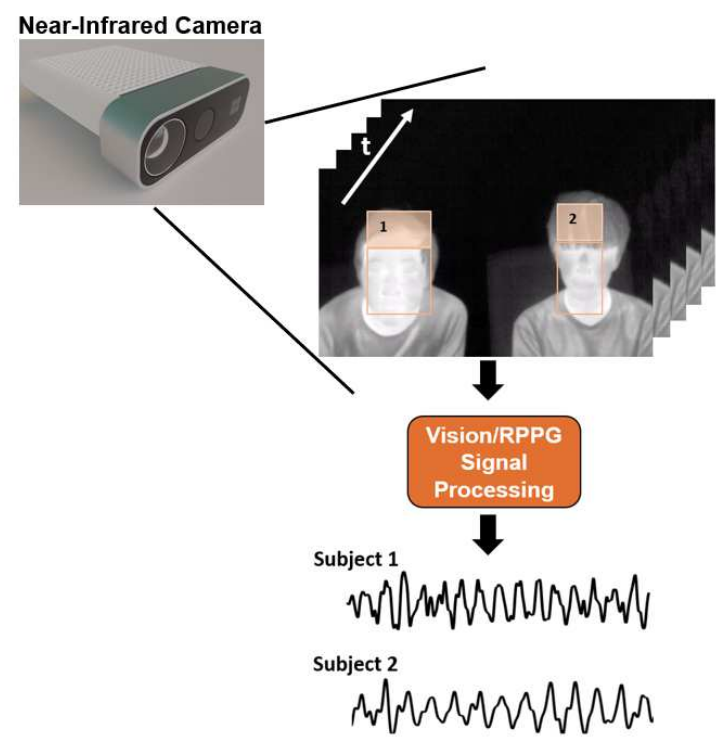

(a)

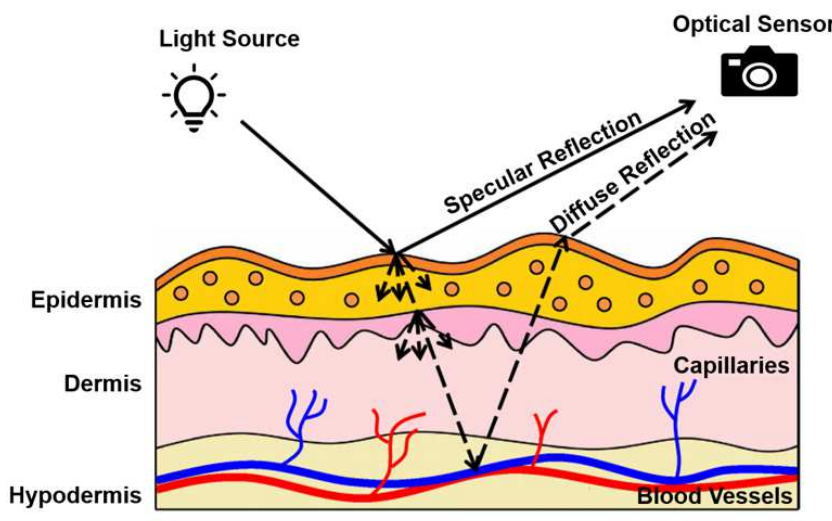

(b)

Figure 5. Working principle of example remote PPG (rPPG) modified from reference ${ }^{56}$. (a) rPPG signal extraction from videos; (b) rPPG principle illustration. 
Table 1. Summary the key features of EM frequencies for vital sign detection.

\begin{tabular}{|c|c|c|c|c|}
\hline \multicolumn{5}{|c|}{ VSM Using EM Waves } \\
\hline Technology & $\begin{array}{l}\text { Microwave } 3,8-15,19,20,30 \\
\text { (a few to a few tens of } \mathrm{GHz} \text { ) }\end{array}$ & $\mathrm{THz}^{22,32-34}(100-10000 \mathrm{GHz})$ & Near-Infrared/Infrared ${ }^{35,36}$ & Visible Light ${ }^{4,37,38}$ \\
\hline Signal Type & Phase, or complex & Magnitude $^{\star}$, phase & Magnitude only & Magnitude only \\
\hline Working Principle & Motion based Doppler effect & $\begin{array}{c}\text { Wave reflectivity* } \\
\text { /motion based Doppler effect }\end{array}$ & Mostly light reflectivity & Most light reflectivity \\
\hline Algorithm Performance & Low phase sensitivity & Good phase sensitivity & Good pulse sensitivity & $\begin{array}{l}\text { Better pulse performance } \\
\text { from more color channels }\end{array}$ \\
\hline Vital Signs Detection & $\begin{array}{l}\text { Breathing dominant } \\
\text { /heartbeat not robust }\end{array}$ & Breathing and heartbeat separable & $\begin{array}{l}\text { Heartbeat detectable } \\
\text { /breathing not robust }\end{array}$ & $\begin{array}{l}\text { Heartbeat detectable } \\
\text { /breathing not robust }\end{array}$ \\
\hline Computation Load & Low & Low & High & High \\
\hline Spatial Resolution & Low (large aperture) & Good (millimeter resolution) & Excellent & Excellent \\
\hline Clutter Performance & Poor & Intermediate & Good & Good \\
\hline Material Penetration (e.g. clothes) & Excellent & Good & Poor & Non-existent \\
\hline $\begin{array}{c}\text { Sensing in Low Visibility } \\
\text { Conditions (e.g. smoke, fog) }\end{array}$ & Excellent & Excellent & Good & Poor \\
\hline $\begin{array}{c}\text { Synergy with Radar Imaging } \\
(\text { e.g. NLOS })\end{array}$ & Poor (coarse imaging) & Excellent & None & None \\
\hline $\begin{array}{c}\text { Cost and } \\
\text { Manufacturing Effort }\end{array}$ & Cheap & Very high & High & Very cheap \\
\hline Privacy Issue & None & None & Mild & Yes \\
\hline
\end{tabular}

$\star, *$ This work.

body movements, which are significantly stronger than the pulse signal in the radar return due to larger radar-cross-section and physical displacement. Direct pulse measurement from chest area is not possible without suppressing respiration motion and naive spectral separation is not sufficient for HR estimation due to the breathing and heartbeat coupling effect ${ }^{15}$. Active motion cancellation techniques considers UWB ${ }^{16,57}$, dual-radars ${ }^{10,18}$ and $\mathrm{RF}$ front end re-design ${ }^{20}$ producing encouraging results but their effectiveness needs further investigations.

Recently a $\mathrm{THz}$ system ${ }^{22}$ is shown for pulse detection at peripheral body sites because of excellent phase sensitivity due to smaller wavelength. Breathing-free pulse measurement is achievable at major peripheral artery sites, such as wrist, with large BW and focusing beam at THz. Furthermore, this study goes one step further and demonstrates in the following sections measurable plethysmographic signals from the face and other body parts in the $\mathrm{THz}$ magnitude response. Therefore, this new observation is named Terahertz-Wave-Plethysmography (TPG). A detailed comparison of VSM using EM waves is tabulated in Table 1.

\section{Terahertz-Wave-Plethysmography (TPG)}

A novel concept of TPG is described in Fig. 6. TPG detects blood volume changes in the dermis layer by measuring the reflectance of THz wave, similar to the PPG principle. According to references ${ }^{24,58,59}$, $\mathrm{THz}$ wave can reach the termis layer through out the peripheral body parts. Similar skin optical properties found in NIR and visible light for plethysmography also found in $\mathrm{THz}$ waves ${ }^{60,61}$, such that $\mathrm{THz}$ interacts with hemoglobin in blood cell. There are measurable differences in the spectra of blood and its components when the hemoglobin content changes in the $\mathrm{THz}$ frequencies. It therefore can be inferred that the pulsatile variation exists in the $\mathrm{THz}$ wave absorption in an illuminated skin area caused by the difference in absorption curves of oxygenated and deoxygenated blood, and thus TPG is possible. In the following, EM simulation and human subject experimental results are presented to validate the proposed theory.

\section{TPG Measurements}

TPG describes THz wave reflectivity measurement and is obtained from the magnitude response of received signal in time domain. TPG and $\mathrm{mD}$ motion, measurement from phase, are simultaneously extracted using the proposed wideband $\mathrm{THz}$ system. Representative TPG and $\mathrm{mD}$ motion measurements along with multiple types of reference measurements are displayed in Fig. 8. In this example, the forehead of the test subject is illuminated by the THz sensing system. It can be concluded that the magnitude variation mostly corresponds to the THz reflectivity change, similar to the PPG principle. On the other hand, the extracted phase variation of the THz return is breathing-motion dominant and it captures the skin surface vibration, inner tissue movement at the top dermis layer due to pulsation and slightly head movement due to breathing activity. Physiological motion is a quasi-periodic narrowband signal. The fundamental frequency signatures such HR and BR change slowly over time. Therefore, in a short processing window, HR and BR can be estimated by inspecting the peak spectral energy location at the proper frequency regions.

To avoid any man-made artifact and maintain purity of the significant signal components, the raw magnitude and phase 
THz-Wave-Plethysmography (TPG)

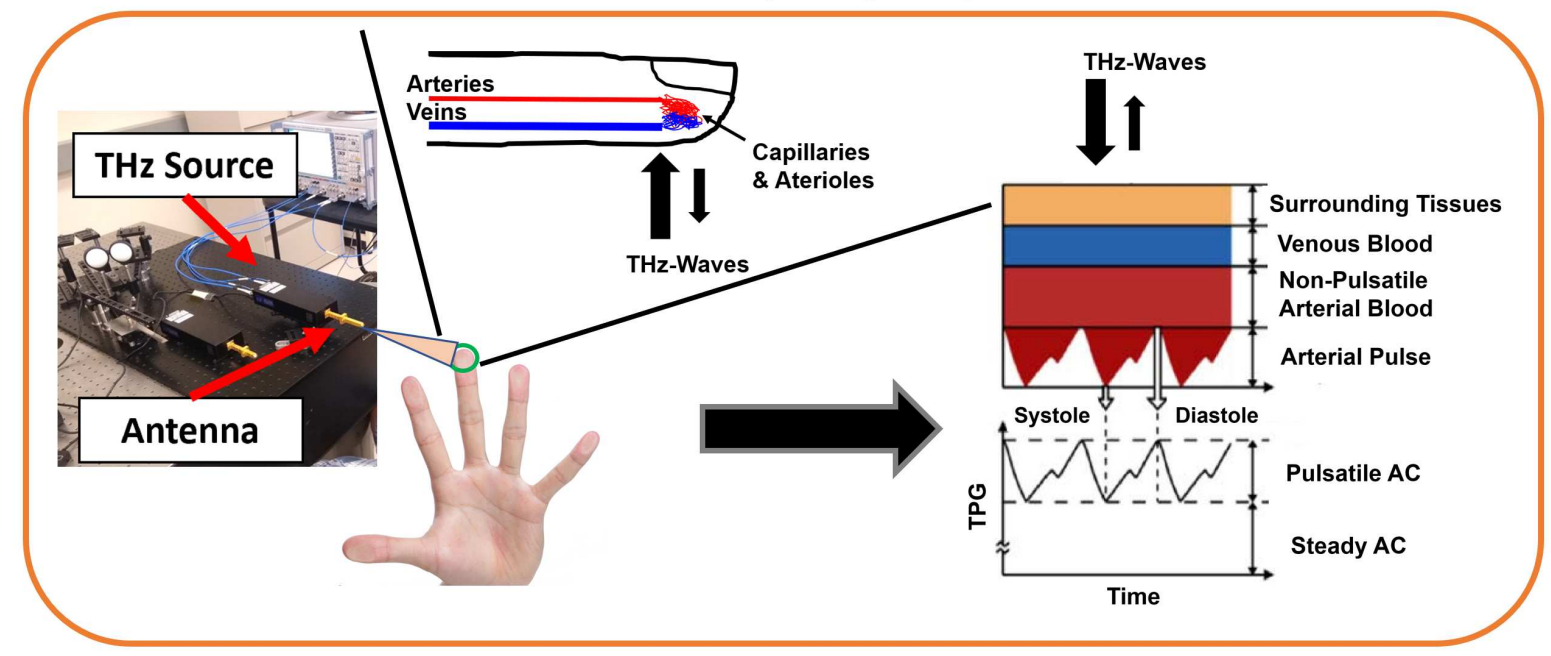

Figure 6. Working principle of THz-Wave-plethysmography (TPG).

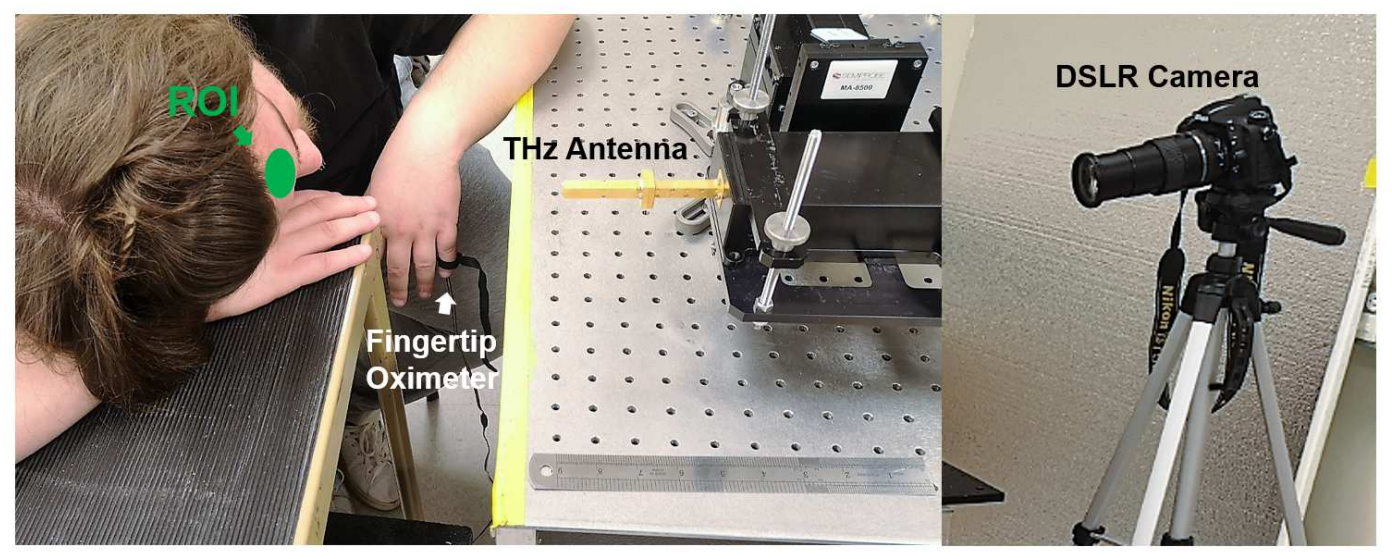

Figure 7. Measurement setup at forehead region of interest (ROI) as indicated in the green area. A fingertip oximeter is used for providing contact PPG signal. Simultaneously, a digital single-lens reflex (DSLR) camera Nikon D750 is focused at the forehead area and recording at 30 frame per second with 1920 x 1080 pixel resolution. The rPPG signal is extracted by processing the sequence of images. 

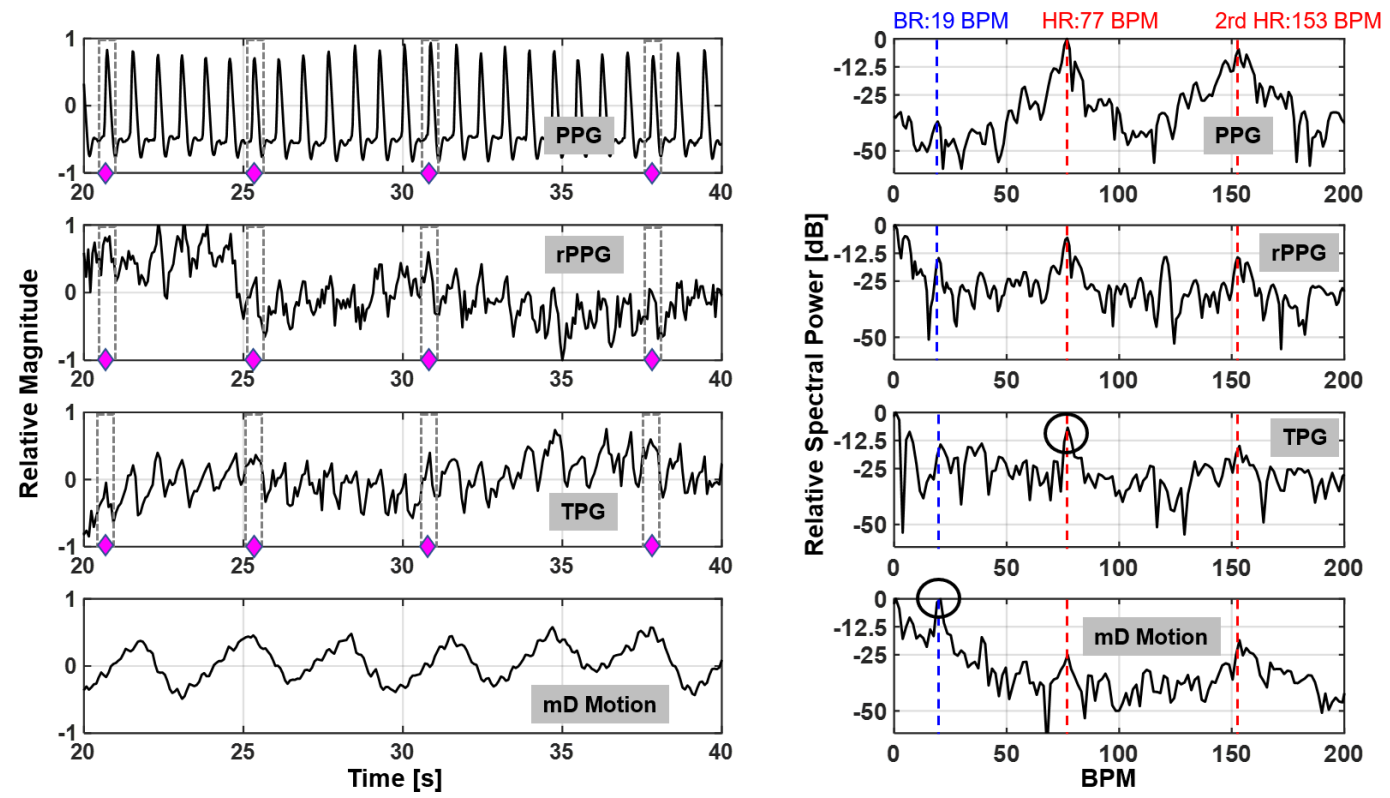

Figure 8. Raw waveform comparison from different measurement sensor outputs and their associated spectra. The left column shows temporal waveform of PPG, rPPG, TPG and $\mathrm{mD}$ phase; the right column their spectra at the frequency of interest. The measurement are synchronized as indicated by the diamond markers. Major spectral components related to pulse and breathing are labeled by blue and red vertical lines. The higher-order harmonic of heartbeat is highlighted for cross-validation of the fundamental heartbeat spectral location.

Contact Approach

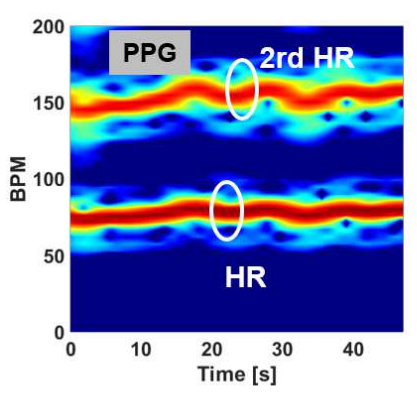

Non-Contact Approaches

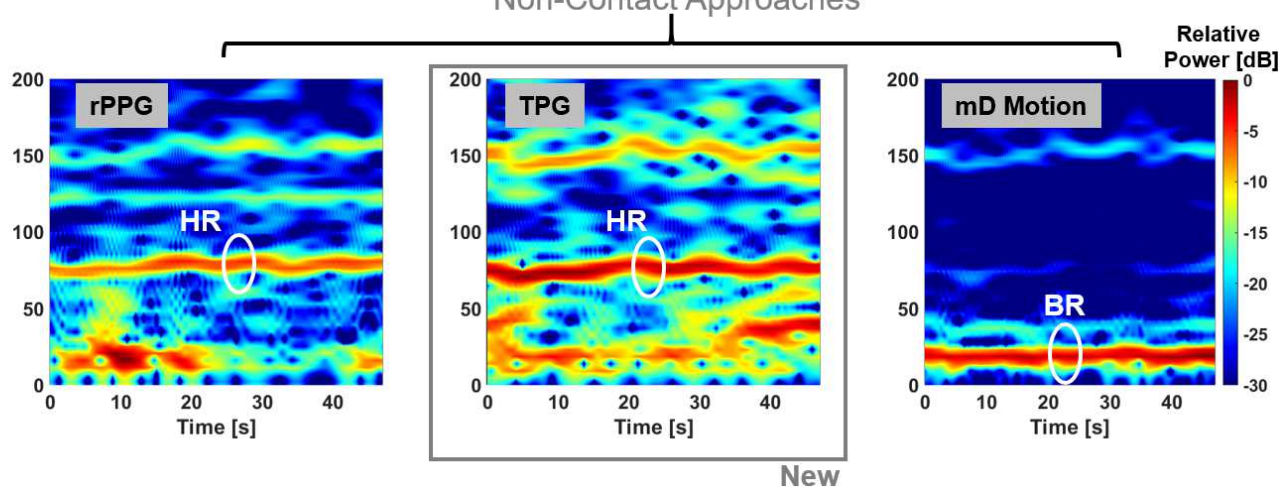

Figure 9. Time-frequency representation of the different measurement data. From the left to the right: spectrograms of PPG, rPPG, TPG, and $\mathrm{mD}$ phase. Based on the operation mode, they are divided into two categories: contact approach, PPG, and non-contact approaches including rPPG, TPG and mD phase. PPG, rPPG and TPG show similar spectral structures, in which the fundamental heartbeat and second-order harmonic of heartbeat are clearly visible and highlighted. The fundamental heartbeat in rPPG and TPG is the most significantly spectral energy. Motion artifacts show up in rPPG and TPG in the form of lower frequency interference close to DC due to random body movement and breathing but they are not dominant. By inspecting the TPG and the mD motion results, it validates the underline principle that the novel TPG measurement is mostly a magnitude-modulated reflectance measurement. 
Table 2. HR Estimation Performance at Peripheral Body Sites

\begin{tabular}{|c|c|c|c|c|c|}
\cline { 2 - 6 } \multicolumn{1}{c|}{} & Palm & Inner Elbow & Temple & Fingertip & Forehead \\
\hline Mean (BPM) & $\mathbf{5 . 2 4}$ & $\mathbf{7 . 0 5}$ & $\mathbf{5 . 8 4}$ & $\mathbf{5 . 4 9}$ & 1.51 \\
\hline std (BPM) & $\mathbf{6 . 9 1}$ & $\mathbf{6 . 6 9}$ & $\mathbf{3 . 7 1}$ & $\mathbf{4 . 7 8}$ & 1.08 \\
\hline
\end{tabular}

information are used to demonstrate the advantages of TPG measurements. The experimental setup is illustrated in Fig. 7, in which the reference signals PPG and rPPG are acquired simultaneously. Raw measurements and their spectra are compared in Fig. 8. The left column represents, from top to bottom, the PPG waveform, the rPPG waveform, the TPG waveform and finally the $\mathrm{mD}$ phase waveform. Minimum processing is applied on each sensor output (scaling) for visualization. The four different types of measurements are aligned. The pulsation signal is the dominant trend in PPG, rPPG and TPG, because they are all reflectivity based measurements. The diamond markers indicate aligned individual pulses in PPG, rPPG, and TPG. While the $\mathrm{mD}$ phase is motion sensitive and is breathing-motion dominant. This observation is consistent with microwave and mmWave radars for $\mathrm{VSM}^{15,30}$. The corresponding vital sign spectra are shown in the right column of Fig.8. No filtering is applied for generating the spectra and only a hanning window is used before taking Fourier transform to suppress sidelobes. Similarly, the major spectral components in PPG, rPPG and TPG are fundamental HR and the $2^{\text {nd }}$-order harmonics of HR. Except strong DC component, the dominant spectral energy in $\mathrm{mD}$ phase is breathing rate (BR). The blue vertical and red vertical lines represent the reference BR and HR and its harmonics. In Fig. 8 second column last row, the highlighted breathing component is about 25 $\mathrm{dB}$ stronger than the possible fundamental pulse component and thus makes it challenging for robust pulse measurement, which is still an open question in microwave radar VSM.

Additionally, the time-frequency analysis is applied to the same dataset. The spectrograms in Fig. 9 are generated using short-time Fourier transform with a sliding window 13-second and one sample increment. The y-axis is the frequency in beats per minute (BPM). An infinite impulse response high pass filter with cut-off frequency $0.1 \mathrm{~Hz}$ is applied in the y-axis direction to remove the zero frequency component (DC) to emphasize the spectral energies of interest. Overall, the contact approach PPG gives best performance, which is used as the standard pulse reference but it requires direct physical contact neutralizing the motivation of remote sensing. On the other hand, the results from the three different non-contract methods provide distinct implications. TPG is similar to the rPPG as the pulse signal almost maintains the stronger spectral components during the experiment. Compared to PPG, rPPG and TPG experience some low frequency interference due to involuntary body motion and breathing motion. These motion artifacts are not constant and dominant thus can be easily separated through post-processing. The $\mathrm{mD}$ phase measurement is known for motion sensitivity and captures breathing motion consistently during the experiment.

Several important observations can be made based on this carefully designed experiments. The breathing signal is not present at the TPG measurement and it validates that the magnitude change originates mostly from the variations in skin conductivity. Plethysmography using $\mathrm{THz}$ wave, therefore, is feasible and our study helps demystify the origin of non-contact reflectance plethysmography. So far the community have not reached consensus on the physical principles of $\mathrm{rPPG}^{62}$. At least two hypotheses on the causes of the observed phenomenon are: 1) optical density change within the tissue caused by arterial pulsations and 2) local deformation of tissue caused by capillaries ${ }^{38}$. Or put it another way, one is EM wave reflectivity change and the other one is local micro-tissue motion. Note that the local micro-tissue motion is a much smaller physical displacement compared the body motion related to respiratory activity, which in this study only shows up in the phase-based (motion sensitive) method. Absence of a stronger breathing component in the TPG waveform and spectrum concludes that the local tissue motion is not the leading cause of the detected pulse in the magnitude response since the large-scale breathing motion is much stronger than the micro-scale local tissue motion.

\section{Accuracy}

The accuracy of TPG measurements is demonstrated at four exemplary body sites: palm, inner elbow, temple and fingertip. These experiments were performed at ASU Terahertz Research Lab. The setups and targeted ROIs are illustrated in Fig. 10. During the experiment, the test subjects were instructed to breath normally and maintain stationary in a relaxing state. However, random body motion and involuntary movements were observed during data acquisition and in reality they are inevitable especially when the experiment time increases.

Four 240-second datasets are used for HR error analysis. The results in Fig. 11 are generated with a sliding window of 13-second with one sample increment. The HR estimation error histogram displays the error distribution at four different levels. The HR estimation performance is calculated as the percentage of measurement points that its estimation error within $(\leq) 20$, $10,5,3$ and 1 BPM respectively. At palm, the measurement error distribution is $94.93 \% \leq 20,89.38 \% \leq 10,72.39 \% \leq 5$, $42.81 \% \leq 3,15.36 \% \leq 1$; inner elbow: $94.12 \% \leq 20,84.97 \% \leq 10,47.71 \% \leq 5,21.24 \% \leq 3,6.54 \% \leq 1$; temple: $99.84 \%$ $\leq 20,86.76 \% \leq 10,45.59 \% \leq 5,23.69 \% \leq 3,8.50 \% \leq 1$; fingertip: $100 \% \leq 20,78.51 \% \leq 10,54.30 \% \leq 5,42.42 \% \leq 3$, $19.12 \% \leq 1$. Overall, on average the error statistics at palm, inner elbow, temple, fingertip and forehead are summarized in 


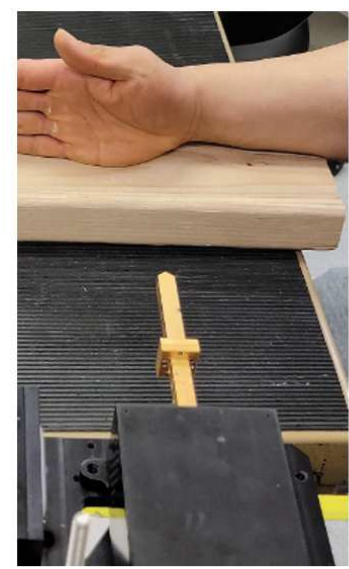

Palm

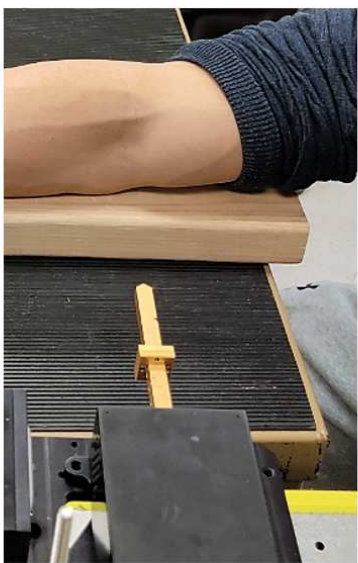

Inner Elbow

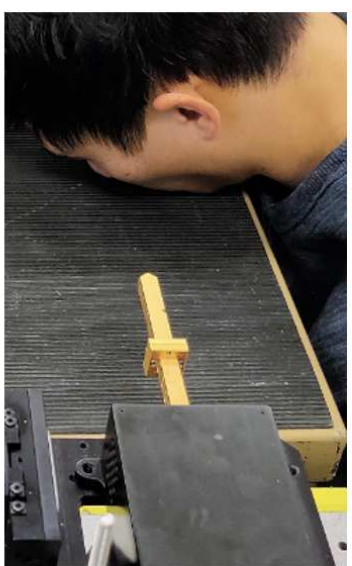

Temple

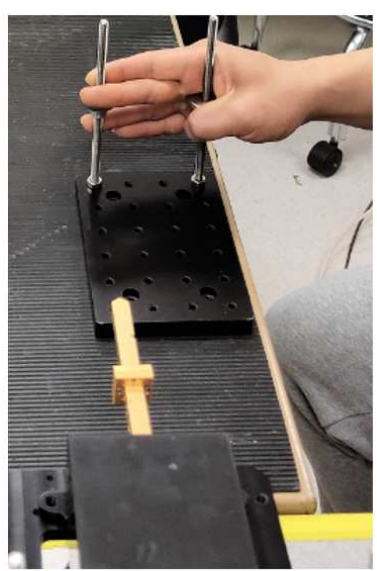

Fingertip

Figure 10. TPG measurement setups at various peripheral body ROIs, including palm, inner elbow, temple and fingertip. (Informed consent is obtained from this test subject for publication of identifying image.)
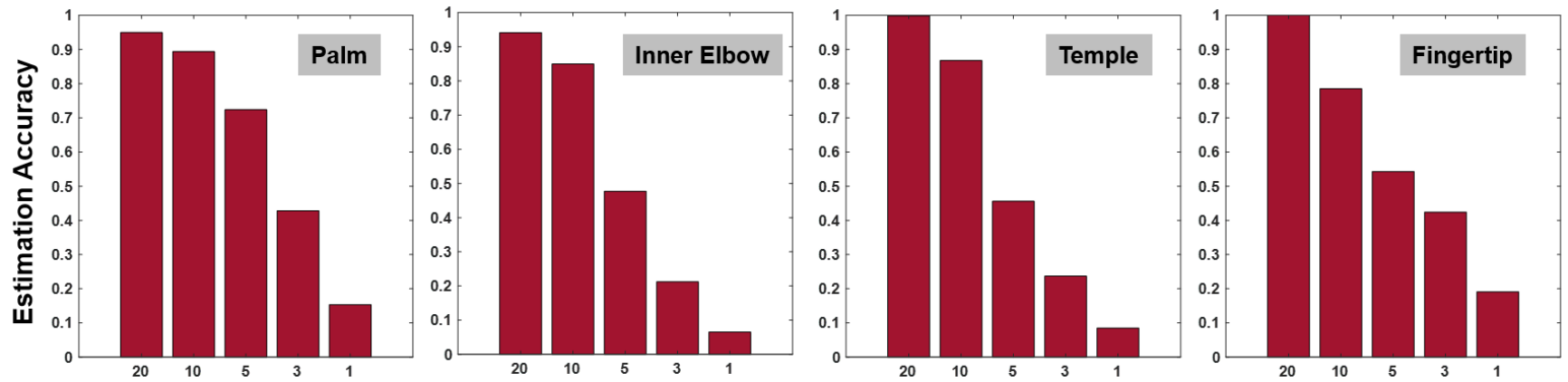

Absolute Error [BPM]

Figure 11. HR estimation error histograms at various peripheral body ROIs in Fig. 10. Each histogram shows the percentage of measurement points that its estimation error within $(\leq)$ 20, 10, 5, 3 and 1 BPM respectively. Palm: $94.93 \% \leq 20,89.38 \% \leq$ $10,72.39 \% \leq 5,42.81 \% \leq 3,15.36 \% \leq 1$; inner elbow: $94.12 \%, 84.97 \%, 47.71 \%, 21.24 \%, 6.54 \%$; temple: $99.84 \%, 86.76 \%$, 45.59\%, 23.69\%, 8.50\%; fingertip: $100 \%, 78.51 \%, 54.30 \%, 42.42 \%, 19.12 \%$; 


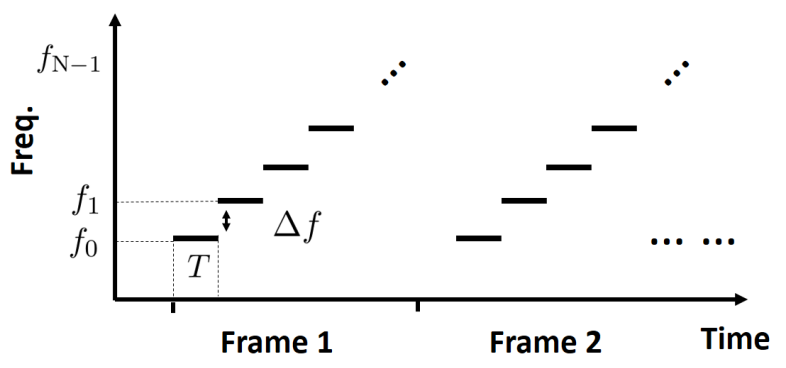

Figure 12. Stepped-FCW radar transmission scheme.

Table 2. HR estimation accuracy from forehead has a mean error $1.51 \mathrm{BPM}$ and std $1.08 \mathrm{BPM}$, and is superior to the other four BOIs because the larger surface area at forehead and better upper body stabilization in prone position as shown in Fig. 7 . These results together validate the feasibility of radar based TPG principle for direct pulse monitoring.

\section{Discussion}

In this study, the feasibility of radar-based plethysmography was investigated using $\mathrm{THz}$ waves. Electrocardiac activities measurement device ECG and contact PPG measurement device are gold standards to measure pulse/HR. The emerging remote sensing technologies using radar and vision sensors transfer the way of measuring the diversity of physiological implications of human body. We have presented a comprehensive review of operating principles and experimental results of the two exciting technologies. For non-disturbance, ubiquitousness, all-weather, penetrability, privacy-preserving sensing requirements, the radar technology is favored in these perspectives. We proposed a novel concept of TPG to extract pulse information analogous to the known optical principle PPG.

$\mathrm{THz}$ radar system detect cardiac pulse based on plethysmography principle in addition to the $\mathrm{mD}$ principle was investigated by a multiplicity of measurements. Exemplary validation measurements show high similarities between radar TPG signal and reference contact-PPG signal regarding the R-peak locations and the spectral peak location. The presented comparison between radar TPG, and $\mathrm{mD}$ motion, rPPG, PPG proves the feasibility of radar-based plethysmography detection. Our analysis considered the differences regarding measurement principles, sizes of the measurement spots, and BOIs. Increased HR estimation error is observed at some BOIs. It is caused by the lower signal-to-noise-ratio (SNR) of the TPG, which can be explained by the surface curvature and area of the measurement spots and measurement stabilization, and which is substantiated by the higher variations of HR estimates. The TPG HR estimation performance can be enhanced by system-level optimization, such as improvement of the dynamic range and emission power of the utilized radar system, and processing optimization. These considerations are not the focus of this study and will be investigated in separate efforts.

Further more, the new insight of cardiac physiology of THz waves interaction with human body at various BOIs improves the direct pulse monitoring performance in a non-contact fashion. The conventional $\mathrm{mD}$ approach focuses at the chest area. It generates noisy and inaccurate signal highly distorted by stronger body movement and breathing motion. Direct pulse monitoring and instantaneous inspection are not feasible using conventional approaches. Recently, research and technology in the field of THz science and electronics has undergone tremendous development, for example THz human body imager ${ }^{23-26}$. Being able to use high spatial resolution THz images to strategically detect pulse information, through clothing or bedding, from multiple spots of human body opens new opportunities for biomedical applications using THz waves: inspecting blood circulation, extracting blood pressure related biometrics such as blood pulse pressure, pulse wave velocity. The unique features of $\mathrm{THz}$ waves, such that they exhibit electron-like and photon-like properties, implies two different ways of VSM. For the first time, radar technology is proven to be able to detect pulse signal using optical principle.

\section{Methods}

\section{Terahertz Sensing System}

The wideband THz sensing setup includes a vector network analyzer (VNA, model: Rohde Schwarz ZVA24) and a frequency extension module (model: Virginia Diodes WR3.4) that up-converts the microwave signals from the VNA to the THz band (220-330 GHz, in which $30 \mathrm{GHz}$ bandwidth is used from 285-315 GHz). A diagonal horn antenna with a gain of $26 \mathrm{~dB}$ is integrated to the extender to emit $\mathrm{THz}$ signals to the free space such that the waves are formed into a narrow beam with a half-power beam width of approximately 10 degrees. As such, the beam illuminates the ROI of human body and the backscattered signals are recorded by measuring the $S_{11}$ parameter. 


\section{Radar Processing}

The system uses a stepped-FCW (SFCW) radar which is an alternative architecture of the UWB radar system and operates in the frequency domain rather than time domain ${ }^{63}$. The SFCW radar transmits a series of discrete narrow band pulses in a stepwise to achieve a larger effective bandwidth. As such, the modulated waveform consists of a group of $N$ coherent pulses with pulse duration $T$, whose frequencies are $f_{\mathrm{n}}=f_{0}+n \Delta f$. Assume that each SFCW waveform has $N$ pulses called one SFCW frame and the center frequency of the first pulse is $f_{0}$, as illustrated in Fig. 12.

One transmitted SFCW frame is represented as a sum of $N$ windowed narrow band signals,

$$
x_{\mathrm{tx}}(\tau)=\frac{1}{\sqrt{T}} \sum_{n} \operatorname{rect}\left(\frac{\tau-n T}{T}\right) \mathrm{e}^{j 2 \pi\left(f_{0}+n \Delta f\right) \tau} .
$$

The backscattered SFCW frame in baseband is modeled by concatenating the down converted received pulses. The received pulse is an attenuated and delayed version of the transmitted pulse at a nominal distance $R_{0}$. However, a slowly time-varying delay is expected due to target motion including body motion related to breathing and surface skin motion related artery pulsation, $R_{T}(t)$ is a function of slow-time $t$,

$$
\tau_{\mathrm{D}}(t)=2 \frac{R_{0}+R_{T}(t)}{c},
$$

where $c$ denotes the speed of light. The fluctuation of vital sign motion caused by breathing and heart beating is modeled as double-harmonics signal. More importantly, another time-varying component exists in $\Gamma\left(t, f_{\mathrm{n}}\right)$ the complex reflection coefficient. In most case $T \gg \tau_{D}(t)$, then the baseband signal can be obtained by down converting and low pass filtering,

$$
x_{\mathrm{rx}}(\tau)=\frac{1}{\sqrt{T}} \sum_{n} \operatorname{rect}\left(\frac{\tau-n T}{T}\right) \Gamma\left(t, f_{\mathrm{n}}\right) \mathrm{e}^{j 2 \pi\left(f_{0}+n \Delta f\right) \tau_{D}(t)} .
$$

The range profile is obtained by performing inverse Fourier transform of the fast time $(\tau)$ frequency samples. The obtained 2-D range slow-time data matrix is ready to be processed. The processing flow is displayed in Fig. 13. First, necessary motion filtering is applied to focus at the range bin of interest. Then, one range bin is selected for simplicity and fixed across the slow-time for further processing to analyze pulse signal. For TPG analysis, the magnitude is extracted by taking the absolute operation of the range located temporal signal while the phasor is extracted by taking angular operation of the same signal. The TPG performance can be further improved and optimized by exploiting multiple range bins in the range profile offered by this wideband THz system. So far, motion-tolerance rPPG is an active research area yet not completely solved. Similarly, the robustness of the TPG method needs further investigation in the presence of motion artifacts such involuntary head motion and this problem will be explored in the future.

\section{EM Simulation Study}

$\mathrm{THz}$ measurements are compared with theoretical data validating the use of reflectivity for the extraction of pulse. The reflectivity-based process is commonly used in the optical spectrum, where an infrared emitter or ambient light illuminates the skin and the intensity of the backscattered lights is modulated ${ }^{38,64,65}$. Using the time-variant magnitude response of the reflected signals, the HR can be extracted.

According to references ${ }^{38,65}$, this modulation in reflectivity has a twofold cause. Firstly, the amount of blood present in the subcutaneous skin vessels and capillaries changes leading to more blood (thus more losses) in the reflected waves. The second cause of this modulation is blood consistency. Namely, the amount of oxygen in the blood varies within the cardiac cycle and the losses of the EM waves are proportional to this variation. For example, the sensitivity of green light radiation to the oxygen levels in the blood is well established enabling the use of green light sensors for the detection of pulse ${ }^{38,64,65}$. However, in a recent study, it was demonstrated using THz spectroscopy that waves ranging from $0.1-1 \mathrm{THz}$ are also sensitive to the consistency of blood ${ }^{61}$. Thus, in this case study, it is assumed that the magnitude modulation in the measurements is attributed to the sub-skin conductivity variation, caused both by the amount of blood in the subcutaneous capillaries and the water concentration in it.

To study the reflectivity modulation caused by the cardiovascular activity at the peripheral body sites, the skin model shown in Fig. 14 is considered. The skin is modeled as a three-layered structure ${ }^{66,67}:$ the top part is the thin layer of the stratum corneum, followed by the epidermis where the presence of capillaries is very limited, and finally the dermis which is modeled as a semi-infinite layer. The EM material properties of these layers are tabulated in Table 3. As such, the stratum corneum and the epidermis have low conductivity and the dermis is a conductive layer since it is filled with capillaries. During the cardiac cycle, the variation of blood volume is assumed to modulate the conductivity of the dermis, leading to the modulation of the reflected $\mathrm{THz}$ waves. 


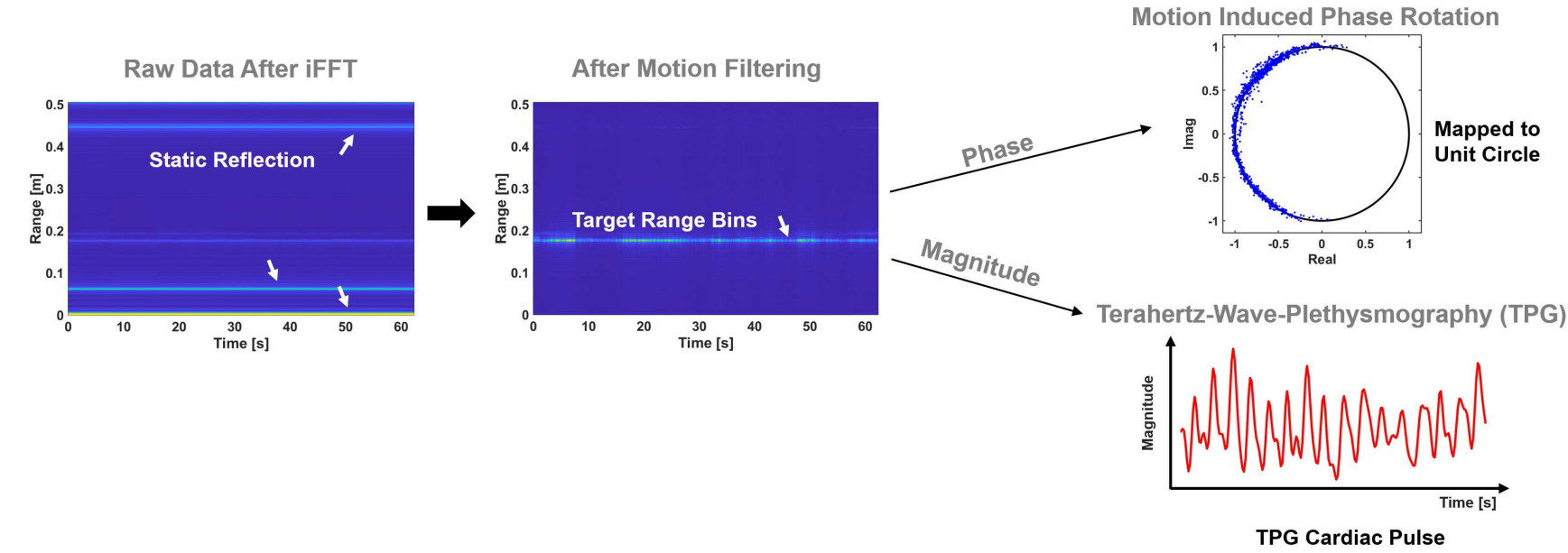

Figure 13. High-level signal processing flow for $\mathrm{THz}$ radar data. From left to the right, they are raw 2-D range slow-time heatmap showing stronger static reflections, motion filtered 2-D heatmap showing only the target response, and the motion measurement extracted from the phase response displayed in the real and imaginary space and lastly the reflectance TPG measurement from the magnitude response.

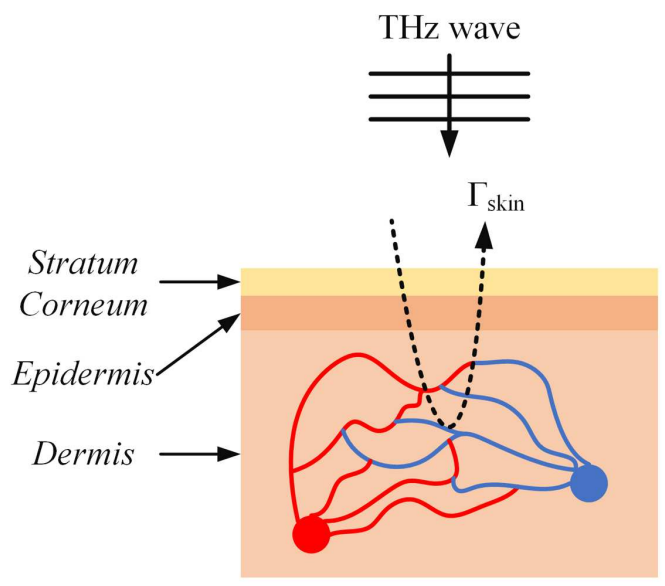

Figure 14. The upper skin structure during the cardiac cycle. In the systole phase, the blood volume is smaller leading to lower tissue conductivity. On the contrary, during the diastole, the arteries and capillaries expand, leading to more blood volume within the tissue, thus higher conductivity.

Table 3. Skin Model Parameters

\begin{tabular}{|c|c|c|c|c|}
\hline & Thickness & $\eta_{r}$ & $\sigma_{\text {diastole }}(\mathrm{S} / \mathrm{m})$ & $\sigma_{\text {systole }}(\mathrm{S} / \mathrm{m})$ \\
\hline Stratum Corneum (sc) & 5 & 2.4 & $10^{-5}$ & $10^{-5}$ \\
\hline Epidermis (ep) & 90 & 3.2 & $\mathbf{1}$ & $\mathbf{1}$ \\
\hline Dermis (derm) & Indefinite-half-space & 3.9 & 41 & 36 \\
\hline
\end{tabular}


The dermis layer conductivity as given in Table 3, is calculated by,

$$
\sigma_{\text {dermis }}=(1-\xi) \sigma_{\text {skin,dry }}+\varepsilon \sigma_{\text {blodd }},
$$

where $\sigma_{\text {skin,dry }}$ is the dry skin conductivity ${ }^{68}, \sigma_{\text {skin, } d r y}$ is the blood conductivity ${ }^{68}$, and $\xi$ is the blood concentration. For this case study, the blood concentration is $60 \%$ during the systole and $70 \%$ during the diastole ${ }^{38}$. Then, the reflection coefficient of the skin is given by ${ }^{69}$,

$$
\Gamma_{\text {skin }}=\frac{r_{1}+r_{2} z_{1}+r_{1} r_{2} r_{3} z_{2}+r_{3} z_{1} z_{2}}{1+r_{1} r_{2} z_{1}+r_{2} r_{3} z_{2}+r_{1} r_{3} z_{1} z_{2}}
$$

where

$$
\begin{aligned}
r_{1} & =\frac{\eta_{s c}-\eta_{a i r}}{\eta_{s c}+\eta_{a i r}} \\
r_{2} & =\frac{\eta_{e p}-\eta_{s c}}{\eta_{e p}+\eta_{s c}} \\
r_{3} & =\frac{\eta_{\text {derm }}-\eta_{e p}}{\eta_{\text {derm }}+\eta_{s c}},
\end{aligned}
$$

are the partial reflection coefficients at the air and the stratum corneum interface, the stratum corneum and the the epidermis interface, and the the epidermis interface and the dermis interface. And,

$$
\begin{aligned}
& z_{1}=e^{-2 i k_{s c} t_{s c}} \\
& z_{2}=e^{-2 i k_{e p} t_{e p}} \\
& z_{3}=e^{-2 i k_{d e r m} t_{d e r m}},
\end{aligned}
$$

are the propagation delay in stratum corneum, epidermis and dermis respectively, where

$$
\begin{aligned}
\eta_{\text {air }} & =\sqrt{\varepsilon_{0}} \\
\eta_{x} & =\sqrt{\varepsilon_{x}\left(1-i \frac{\sigma_{x}}{2 \pi f \varepsilon_{x}}\right)},
\end{aligned}
$$

and

$$
k_{x}=\frac{2 \pi f \sqrt{\varepsilon_{x}}}{c}, x=s c, e p, \text { or derm } .
$$

where the subscript $x$ denotes either the stratum corneum, the epidermis, or the dermis, $f$ the frequency, $\varepsilon_{0}$ the free space permittivity, and $c$ the speed of light in free space.

The aforementioned equations define the reflection coefficient under plane wave illumination for a specific value of $\xi$. However, the value of the $\xi$ can vary between $60 \%$ to $70 \%$ during the cardiac cycle ${ }^{38}$. Thus, the $\xi$ of (2) is defined as

$$
\xi=0.65+0.05 \cos (2 \pi \alpha t / 60),
$$

where $t$ is the time in seconds and $\alpha$ denotes HR. As such, for every time instance within the cardiac cycle, a different $\xi$ value is calculated and the respective reflection coefficient $\left(\Gamma_{\text {skin }}\right)$ is computed using (2)-(11). The reflection coefficient can be calculated for various values of $t$ and $f$, creating a $2 \mathrm{D}$ vector $\Gamma_{\text {skin }}(f, t)$. This complex set of values are then used to carry out the computation of the HR using the TPG algorithm, by using the magnitude of the complex reflection coefficient for every $(f, t)$ pair.

TPG frequency spectra are displayed in Fig. 15a-c. The skin's reflection coefficient is calculated in the 285-315 GHz range with a step of $214.3 \mathrm{MHz}$ and a HR of $72 \mathrm{BPM}$, for various time sampling rates. As shown, the sampling rate does not affect the two peaks produced by the TPG algorithm, which correspond to the fundamental and $2^{\text {nd }}$-order harmonic of the HR, respectively. The frequency separation between the first and the second peak in the TPG spectra varies as the HR changes in Fig. 15d-f, thus validating that the second peak in the TPG spectra is a harmonic of the HR, produced by the non-linearity of (3). The proposed mathematical model, is further validated by the results presented in Fig. 16. The magnitude of the calculated $\Gamma_{\text {skin }}$ in the $285-315 \mathrm{GHz}$ range within a 20 second window and a HR of 72 BPM is depicted in Fig. 16(a). Sampling time and duration of the numerical experiment are the same as the one used in the measurement campaign. The simulated pulse 


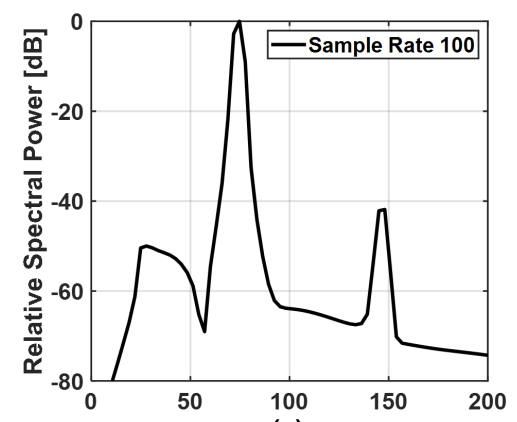

(a)

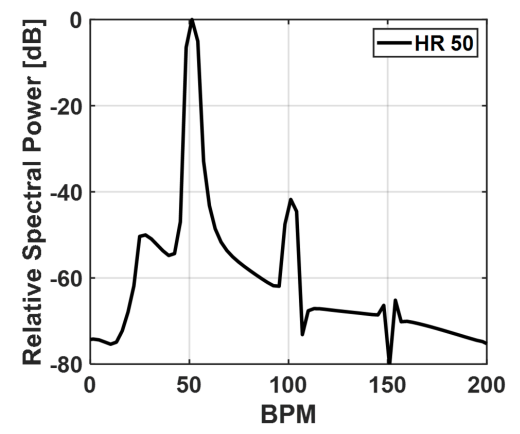

(d)

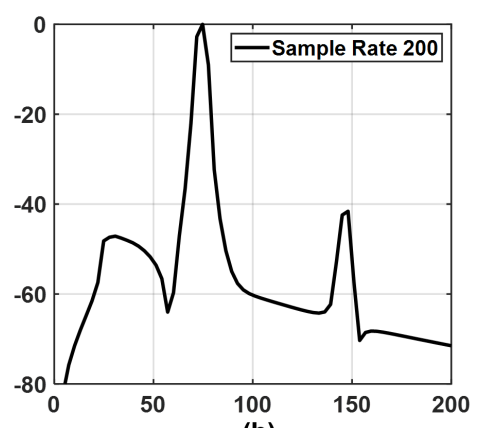

(b)

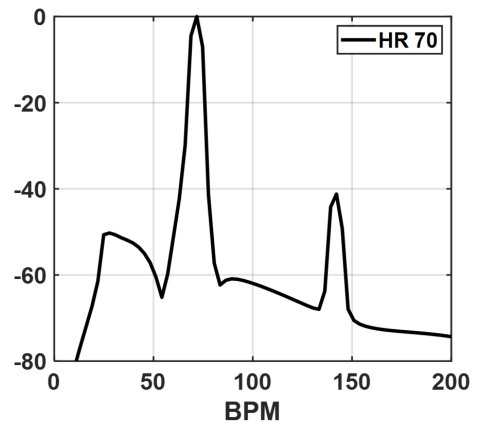

(e)

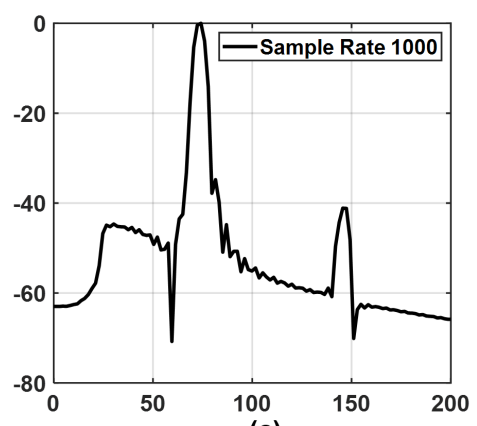

(c)

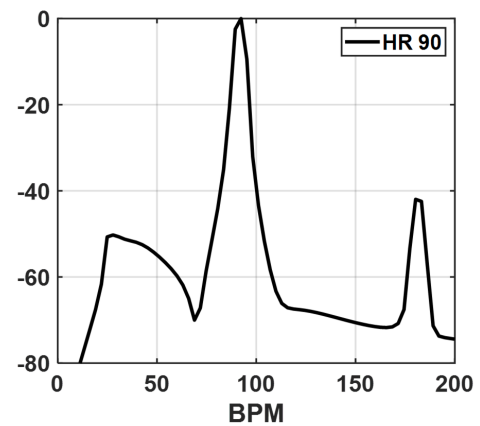

(f)

Figure 15. Spectrum from simulated data as function of sample rate and blood flow cycles. The first row (a)-(c) shows the spectra from sample rate (samples/second) at 100, 200 and 1000 with blood flow cycles fixed at 72 cycles per minute; the second row (d)-(f) the spectra at 50 cycles, 70 cycles, 90 cycles with a fix sample rate of 100.

waveforms and spectra are shown in Fig. 16(b)-(c), and the corresponding measurements in Fig. 16(d)-(e). As such, the peak-to-valley modulation on the THz reflection coefficient during the cardiac cycle is close to $1.8 \mathrm{~dB}$ in Fig. 16(b), which is in agreement with the THz measurements presented in Fig. 16(d). The THz measurement in Fig. 16(d) is post-processed by applying a bandpass filter to remove the static DC component and lower frequency component. The agreement is also found spectrally by inspecting the pulse frequency energy concentration in Fig. 16(c)(e). Therefore, above theoretical approach verifies that the blood concentration in the upper layers of the skin, during the cardiac cycle, leads to the measurable reflectivity change in the $\mathrm{THz}$ frequency range. This effect enables pulse detection using the $\mathrm{THz}$ reflectivity measurements, which are not dominated by the breathing motions and other body motions, thus, leading to a robust pulse monitoring tool.

\section{Pulse Sensitivity Study in TPG and rPPG Measurements}

Forehead BOI is considered for pulse sensitivity analysis. The experimental setup is shown in Fig. 17. TPG and rPPG measurements are taken simultaneously from the forehead of the test subject. One snapshot of the heartbeat spectral result is shown in Fig. 18(a) TPG and 18(b) rPPG. Red curve indicates the reference PPG spectrum. The pulse measurement quality is greater in this TPG example. Pulse-signal-to-interference-plus-noise-ratio (PSINR) is used to quantitatively evaluate the pulse sensitivity. The PSINR is calculated as the ratio of the area under the power spectrum curve in a region surrounding the maximum peak in the frequency domain, to the area under the curve in the HR frequency range of interest, as illustrated in Fig. 18(a). The calculated PSINR of the TPG measurement is $-1.34 \mathrm{~dB}$ and $-5.48 \mathrm{~dB}$ for the rPPG measurement. The lower PSINR value (or poorer pulse sensitivity) leads to less accurate HR estimates over time. Further more, empirical CDF of HR estimation error is used to analyze the relationship between estimation performance and pulse sensitivity. Fig.18(c) is obtained from a 180-second dataset. The HR is estimated from the spectral peak location. The area under the empirical CDF curve for the TPG measurement is larger than that of the rPPG measurement, and therefore better estimation performance.

In this study, the TPG outperforms the rPPG performance in terms of PSINR and CDF. The poorer HR estimation performance in rPPG can be explained by unfavored lighting condition (no dedicated lighting is applied for rPPG measurement) and further measurement distance between the camera and the test subject (about 2 meters). Though it is not strictly a fair comparison between TPG and rPPG for HR estimation performance, it again highlights the usage of THz wave as non-contact reflectance method for probing cardiac pulse, which was only proven in optical waves using rPPG principle. The reported TPG and rPPG HR estimation performance can be improved further with respect to the experiment setup and algorithmic 


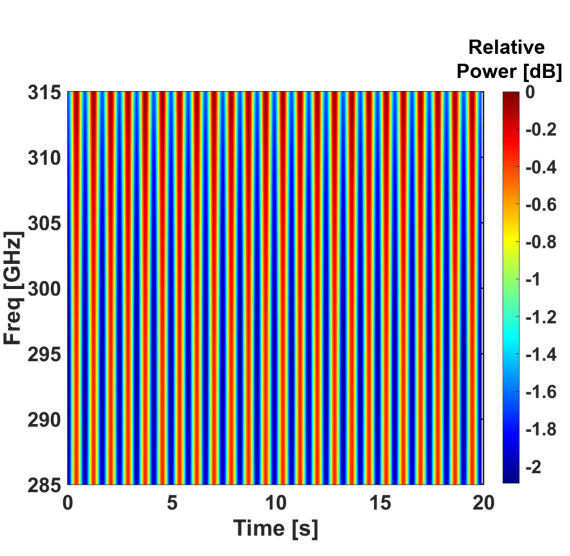

(a) Simulated skin reflection coefficients

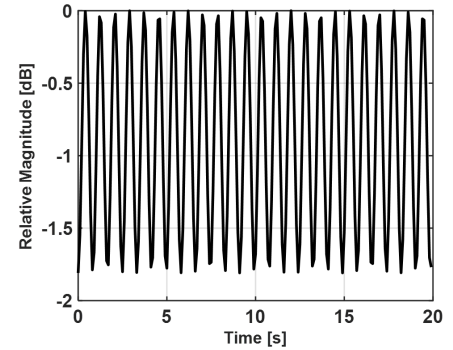

(b) Simulated pulse waveform

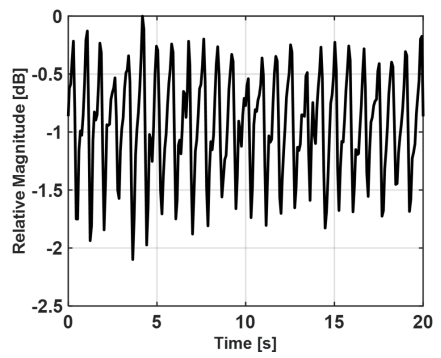

(d) Measured pulse waveform

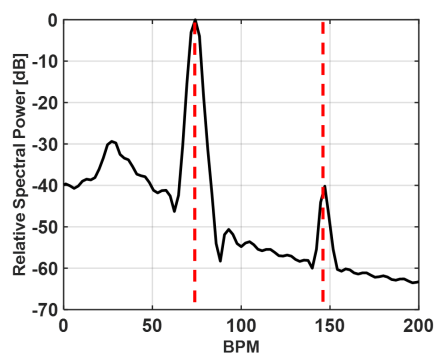

(c) Simulated pulse spectrum

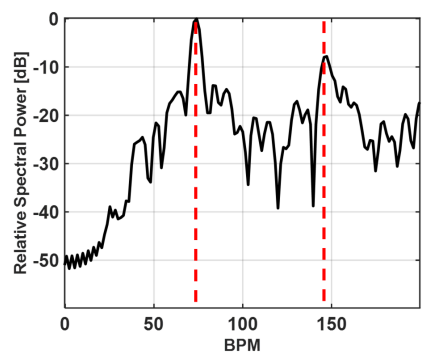

(e) Measured pulse spectrum

Figure 16. (a) The simulated skin reflection coefficients from time-varying dermis conductivity values due to cyclical blood flow (computer simulated at 72 cycles per minute) in the $285-315 \mathrm{GHz}$ spectrum; (b) the corresponding pulse waveform extracted by averaging the simulated skin reflection coefficients across the frequency range; (c) the corresponding pulse frequency spectrum of the simulated data; (d) the reference THz pulse measurement in $285-315 \mathrm{GHz}$ spectrum taken from a subject's forehead; (d) the corresponding pulse frequency spectrum of the measured data. The peak-to-valley variation of the measurements (d) is similar to the one observed by the simulated data (b) approximately $1.8 \mathrm{~dB}$ and also the fundamental and second-order heartbeat harmonics locations are matched in the simulation data (c) and the measurement data (e), thus validating the working principle of the novel TPG measurement approach.

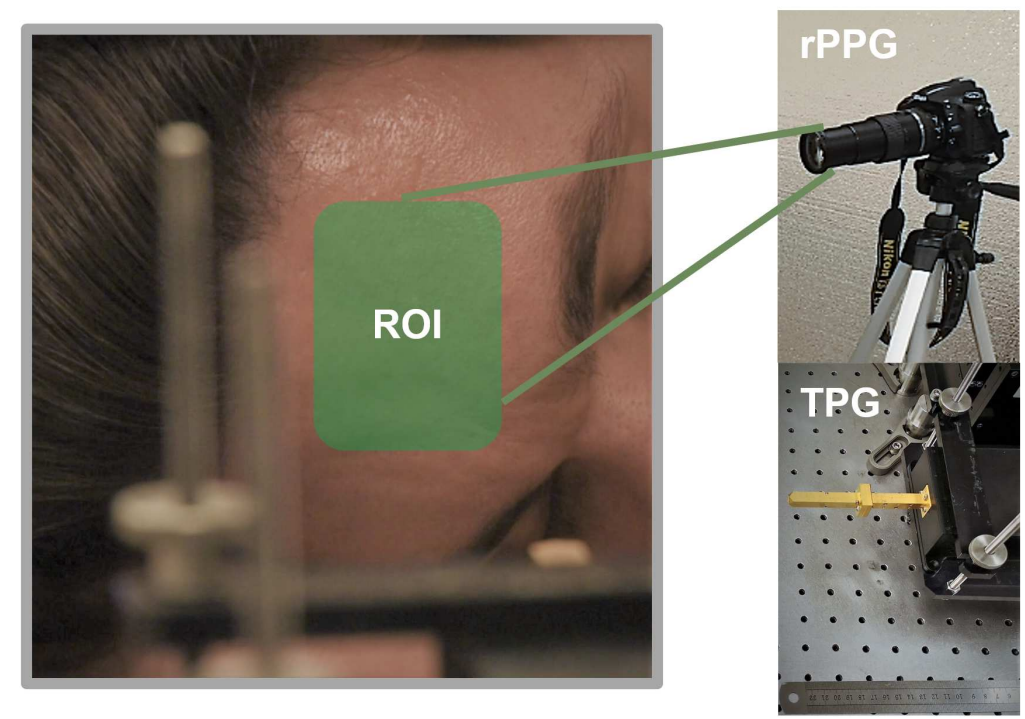

Figure 17. One Snapshot from the recorded videos, focusing on forehead of the test subject. The shaped area in the left figure is the manually selected area to extract rPPG signals in Fig. 18(b) from the sequence of image frames. rPPG measurement and TPG measurement setups are displayed in the right figure. (Informed consent is obtained from this test subject for publication of identifying image.) 


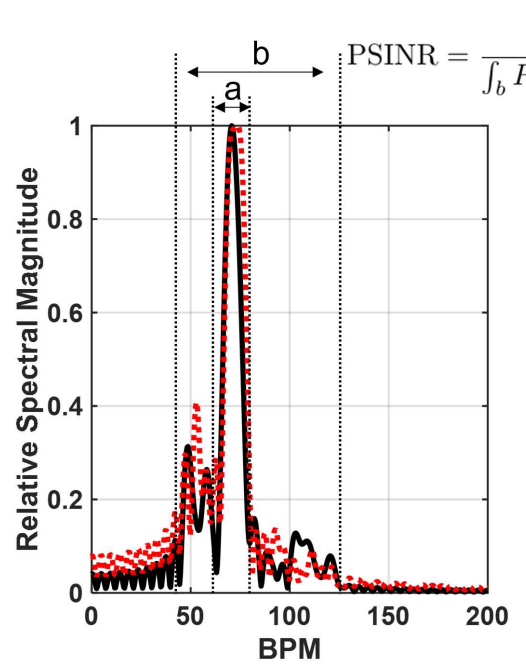

(a) TPG

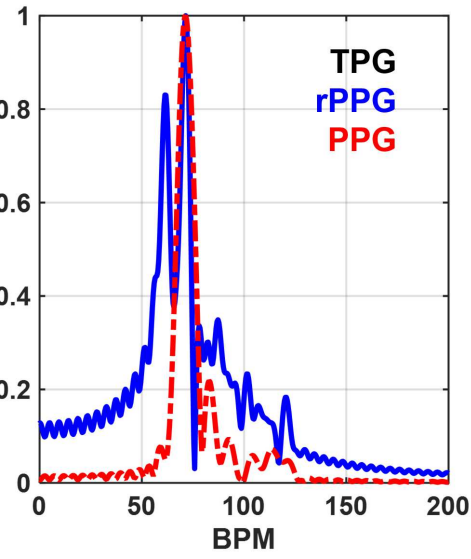

(b) rPPG

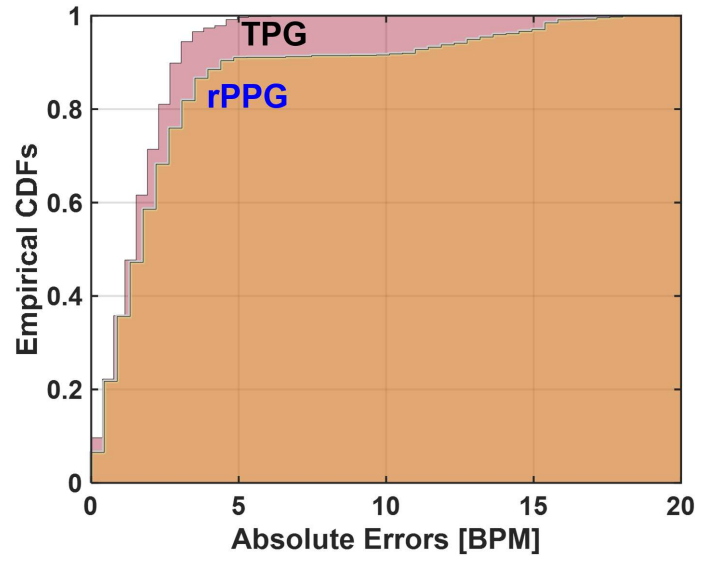

(c) CDF comparison

Figure 18. Pulse sensitivity study in the TPG and the rPPG measurements.(a)(b) Visualization of pulse sensitivity in the TPG and the rPPG, and quantification of pulse sensitivity using the PSINR metric; (c) Comparison of HR estimation performance in the form of empirical CDF from the TPG and the rPPG.

development. However, these efforts are out of scope of this paper and will be investigated in future endeavors.

\section{Subjects and Experimental Protocol}

Measurements were taken from 4 human test subjects. Totally our database contains 8540 seconds of data, comprising asynchronized raw data of PPG, rPPG and data derived from radar. All measurements were recorded under standardized conditions, seated comfortably in an arm chair with back support and breathing normally at leisure. During the experiments, the distance between antenna and BOIs varied from 10 centimeters to 60 centimeters. Additionally, predefined interventions were considered, changing measurement positions including sitting, standing and lying down, changing heartbeat variability by physical exercising for 5 minutes before measurements, changing breathing pattern by holding breathing for 15 seconds to 30 seconds. Data acquisition was acquired following the study protocol over different BOIs, such as finger, forehead, inner elbow, which are illustrated in Fig. 10.

\section{Data Availability}

The data that support the findings of this study are available from the corresponding authors upon reasonable request.

\section{Human Subjects}

The study was approved by the ethics committee of the Arizona State University. All research was performed in accordance with relevant guidelines and regulations. The informed consent was obtained from all subjects in human trials.

\section{References}

1. Chen, V. C. \& Ling, H. Time-frequency transforms for radar imaging and signal analysis (Artech house, 2002).

2. Chen, V. C., Li, F., Ho, S.-S. \& Wechsler, H. Micro-doppler effect in radar: phenomenon, model, and simulation study. IEEE Transactions on Aerosp. electronic systems 42, 2-21 (2006).

3. Lin, J. C. Noninvasive microwave measurement of respiration. Proc. IEEE 63, 1530-1530 (1975).

4. Poh, M.-Z., McDuff, D. J. \& Picard, R. W. Non-contact, automated cardiac pulse measurements using video imaging and blind source separation. Opt. express 18, 10762-10774 (2010).

5. Miyakawa, M. Tomographic measurement of temperature change in phantoms of the human body by chirp radar-type microwave computed tomography. Med. \& biological engineering \& computing 31, S31-S36 (1993).

6. Meaney, P., Paulsen, K., Fanning, M., Li, D. \& Fang, Q. Image accuracy improvements in microwave tomographic thermometry: phantom experience. Int. journal hyperthermia 19, 534-550 (2003). 
7. Klemm, M., Craddock, I., Leendertz, J., Preece, A. \& Benjamin, R. Breast cancer detection using symmetrical antenna array. In The Second European Conference on Antennas and Propagation, EuCAP 2007, 1-5 (IET, 2007).

8. Immoreev, I. \& Tao, T.-H. Uwb radar for patient monitoring. IEEE Aerosp. Electron. Syst. Mag. 23, 11-18 (2008).

9. Wang, F.-K. et al. Detection of concealed individuals based on their vital signs by using a see-through-wall imaging system with a self-injection-locked radar. IEEE Transactions on Microw. Theory Tech. 61, 696-704 (2013).

10. Li, C. \& Lin, J. Complex signal demodulation and random body movement cancellation techniques for non-contact vital sign detection. In Microwave Symposium Digest, 2008 IEEE MTT-S International, 567-570 (IEEE, 2008).

11. Park, B.-K., Boric-Lubecke, O. \& Lubecke, V. M. Arctangent demodulation with dc offset compensation in quadrature doppler radar receiver systems. IEEE transactions on Microw. theory techniques 55, 1073-1079 (2007).

12. Singh, A. et al. Data-based quadrature imbalance compensation for a cw doppler radar system. IEEE Transactions on Microw. Theory Tech. 61, 1718-1724 (2013).

13. Guan, S., Rice, J. A., Li, C. \& Gu, C. Automated dc offset calibration strategy for structural health monitoring based on portable cw radar sensor. IEEE Transactions on Instrumentation Meas. 63, 3111-3118 (2014).

14. Ren, L., Wang, H., Naishadham, K., Kilic, O. \& Fathy, A. E. Phase-based methods for heart rate detection using uwb impulse doppler radar. IEEE Transactions on Microw. Theory Tech. 64, 3319-3331 (2016).

15. Rong, Y. \& Bliss, D. W. Remote sensing for vital information based on spectral-domain harmonic signatures. IEEE Transactions on Aerosp. Electron. Syst. (2019).

16. Rong, Y., Chiriyath, A. R., Dutta, A. \& Bliss, D. W. Active breathing suppression for improved sleep monitoring heartbeat detection using uwb radar. In 2019 IEEE 8th International Workshop on Computational Advances in Multi-Sensor Adaptive Processing (CAMSAP), 161-165 (IEEE, 2019).

17. Rong, Y., Mishra, K. V. \& Bliss, D. W. Multiple moving targets heartbeat estimation and recovery using multi-frequency radars. In 2021 IEEE Radar Conference (RadarConf21), 1-5 (IEEE, 2021).

18. Rong, Y., Dutta, A., Chiriyath, A. \& Bliss, D. W. Motion-tolerant non-contact heart-rate measurements from radar sensor fusion. Sensors 21, 1774 (2021).

19. Lazaro, A., Girbau, D. \& Villarino, R. Analysis of vital signs monitoring using an ir-uwb radar. Prog. In Electromagn. Res. 100, 265-284 (2010).

20. Tang, M.-C., Kuo, C.-Y., Wun, D.-C., Wang, F.-K. \& Horng, T.-S. A self-and mutually injection-locked radar system for monitoring vital signs in real time with random body movement cancellation. IEEE Transactions on Microw. Theory Tech. 64, 4812-4822 (2016).

21. Gu, C., Wang, G., Li, Y., Inoue, T. \& Li, C. A hybrid radar-camera sensing system with phase compensation for random body movement cancellation in doppler vital sign detection. IEEE Transactions on Microw. Theory Tech. 61, 4678-4688 (2013).

22. Rong, Y., Theofanopoulos, P. C., Trichopoulos, G. C. \& Bliss, D. W. Cardiac sensing exploiting an ultra-wideband terahertz sensing system. In 2020 IEEE International Radar Conference (RADAR), 1002-1006 (IEEE, 2020).

23. Theofanopoulos, P. C., Sakr, M. \& Trichopoulos, G. C. Multistatic terahertz imaging using the radon transform. IEEE Transactions on Antennas Propag. 67, 2700-2709 (2019).

24. Theofanopoulos, P. C. \& Trichopoulos, G. C. A terahertz microscopy technique for sweat duct detection. In 2018 IEEE/MTT-S International Microwave Symposium-IMS, 864-867 (IEEE, 2018).

25. Cooper, K. B. et al. Thz imaging radar for standoff personnel screening. IEEE Transactions on Terahertz Sci. Technol. 1, 169-182 (2011).

26. Gao, J. et al. Fast three-dimensional image reconstruction of a standoff screening system in the terahertz regime. IEEE Transactions on Terahertz Sci. Technol. 8, 38-51 (2017).

27. Theofanopoulos, P. C. \& Trichopoulos, G. C. A novel 2-bit graphene reconfigurable reflectarray. In 2020 IEEE International Symposium on Antennas and Propagation and North American Radio Science Meeting, 1701-1702 (IEEE, 2020).

28. Kashyap, B. G., Theofanopoulos, P. C., Cui, Y. \& Trichopoulos, G. C. Mitigating quantization lobes in mmwave low-bit reconfigurable reflective surfaces. IEEE Open J. Antennas Propag. 1, 604-614 (2020).

29. Theofanopoulos, P. \& Trichopoulos, G. C. A single-switch-per-bit topology for mmwave and thz reconfigurable reflective surfaces. (2021). 
30. Ahmad, A., Roh, J. C., Wang, D. \& Dubey, A. Vital signs monitoring of multiple people using a fmcw millimeter-wave sensor. In 2018 IEEE Radar Conference (RadarConf18), 1450-1455 (IEEE, 2018).

31. Alizadeh, M., Shaker, G., De Almeida, J. C. M., Morita, P. P. \& Safavi-Naeini, S. Remote monitoring of human vital signs using mm-wave fmcw radar. IEEE Access 7, 54958-54968 (2019).

32. Petkie, D. T. et al. Remote respiration and heart rate monitoring with millimeter-wave/terahertz radars. In Millimetre Wave and Terahertz Sensors and Technology, vol. 7117, 71170I (International Society for Optics and Photonics, 2008).

33. Benton, C., Bryan, E. \& Petkie, D. T. Terahertz radar for remote measurement of vital signs. Bull. Am. Phys. Soc. 53 (2008).

34. Petkie, D. T., Benton, C. \& Bryan, E. Millimeter-wave radar for vital signs sensing. In Radar Sensor Technology XIII, vol. 7308, 73080A (International Society for Optics and Photonics, 2009).

35. Zhang, Q., Zhou, Y., Song, S., Liang, G. \& Ni, H. Heart rate extraction based on near-infrared camera: Towards driver state monitoring. IEEE Access 6, 33076-33087 (2018).

36. Nowara, E. M., Marks, T. K., Mansour, H. \& Veeraraghavany, A. Sparseppg: towards driver monitoring using camera-based vital signs estimation in near-infrared. In 2018 IEEE/CVF Conference on Computer Vision and Pattern Recognition Workshops (CVPRW), 1353-135309 (IEEE, 2018).

37. Poh, M.-Z., McDuff, D. J. \& Picard, R. W. Advancements in noncontact, multiparameter physiological measurements using a webcam. IEEE transactions on biomedical engineering 58, 7-11 (2010).

38. Kamshilin, A. A. et al. A new look at the essence of the imaging photoplethysmography. Sci. reports 5, 10494 (2015).

39. Churkin, S. \& Anishchenko, L. Millimeter-wave radar for vital signs monitoring. In 2015 IEEE International Conference on Microwaves, Communications, Antennas and Electronic Systems (COMCAS), 1-4 (IEEE, 2015).

40. Feng, L., Po, L.-M., Xu, X., Li, Y. \& Ma, R. Motion-resistant remote imaging photoplethysmography based on the optical properties of skin. IEEE Transactions on Circuits Syst. for Video Technol. 25, 879-891 (2014).

41. Chen, K.-M., Misra, D., Wang, H., Chuang, H.-R. \& Postow, E. An x-band microwave life-detection system. IEEE transactions on biomedical engineering 697-701 (1986).

42. Chen, K.-M., Huang, Y., Zhang, J. \& Norman, A. Microwave life-detection systems for searching human subjects under earthquake rubble or behind barrier. IEEE transactions on biomedical engineering 47, 105-114 (2000).

43. Rong, Y. \& Bliss, D. W. Is radar cardiography (rcg) possible? In Radar Conference (RadarConf), 2019 IEEE (IEEE, 2019).

44. Li, C. \& Lin, J. Random body movement cancellation in doppler radar vital sign detection. IEEE Transactions on Microw. Theory Tech. 56, 3143-3152 (2008).

45. Li, C., Xiao, Y. \& Lin, J. Experiment and spectral analysis of a low-power $k a$-band heartbeat detector measuring from four sides of a human body. IEEE Transactions on Microw. Theory Tech. 54, 4464-4471 (2006).

46. Tang, M.-C., Wang, F.-K. \& Horng, T.-S. Single self-injection-locked radar with two antennas for monitoring vital signs with large body movement cancellation. IEEE Transactions on Microw. Theory Tech. 65, 5324-5333 (2017).

47. Biswas, D., Simões-Capela, N., Van Hoof, C. \& Van Helleputte, N. Heart rate estimation from wrist-worn photoplethysmography: A review. IEEE Sensors J. 19, 6560-6570 (2019).

48. Kamal, A., Harness, J., Irving, G. \& Mearns, A. Skin photoplethysmography—a review. Comput. methods programs biomedicine 28, 257-269 (1989).

49. Aoyagi, T. \& Miyasaka, K. Pulse oximetry: its invention, contribution to medicine, and future tasks. Anesth. analgesia 94, S1-S3 (2002).

50. Allen, J. Photoplethysmography and its application in clinical physiological measurement. Physiol. measurement 28, R1 (2007).

51. Anderson, R. R., Parrish, J. A. et al. The optics of human skin. J. investigative dermatology 77, 13-19 (1981).

52. Damianou, D. The wavelength dependence of the photoplethysmogram and its implication to pulse oximetry. Ph.D. thesis, University of Nottingham (1995).

53. Verkruysse, W., Svaasand, L. O. \& Nelson, J. S. Remote plethysmographic imaging using ambient light. Opt. express 16, 21434-21445 (2008). 
54. Davila, M. I., Lewis, G. F. \& Porges, S. W. The physiocam: cardiac pulse, continuously monitored by a color video camera. J. Med. Devices 10 (2016).

55. Rong, Y. \& Bliss, D. W. Smart homes: See multiple heartbeats through wall using wireless signals. In 2019 IEEE Radar Conference (RadarConf), 1-6 (IEEE, 2019).

56. Rongt, Y. et al. Respiration and cardiac activity sensing using 3-d cameras. In 2020 54th Asilomar Conference on Signals, Systems, and Computers, 955-959 (IEEE, 2020).

57. Rong, Y., Gutierrez, R., Mishra, K. V. \& Bliss, D. W. Noncontact vital sign detection with uav-borne radars: An overview of recent advances. IEEE Veh. Technol. Mag. (2021).

58. Hayut, I. et al. The helical structure of sweat ducts: Their influence on the electromagnetic reflection spectrum of the skin. IEEE Transactions on Terahertz Sci. technology 3, 207-215 (2012).

59. Tripathi, S. R., Miyata, E., Ishai, P. B. \& Kawase, K. Morphology of human sweat ducts observed by optical coherence tomography and their frequency of resonance in the terahertz frequency region. Sci. reports 5, 9071 (2015).

60. Laman, N., Harsha, S. S., Grischkowsky, D. \& Melinger, J. S. High-resolution waveguide thz spectroscopy of biological molecules. Biophys. journal 94, 1010-1020 (2008).

61. Reid, C. B., Reese, G., Gibson, A. P. \& Wallace, V. P. Terahertz time-domain spectroscopy of human blood. IEEE journal biomedical health informatics 17, 774-778 (2013).

62. Sun, Y., Hu, S., Azorin-Peris, V., Kalawsky, R. \& Greenwald, S. E. Noncontact imaging photoplethysmography to effectively access pulse rate variability. J. biomedical optics 18, 061205 (2012).

63. Noon, D. A. Stepped-frequency radar design and signal processing enhances ground penetrating radar performance. (1996).

64. Humphreys, K., Ward, T. \& Markham, C. Noncontact simultaneous dual wavelength photoplethysmography: a further step toward noncontact pulse oximetry. Rev. scientific instruments 78, 044304 (2007).

65. Spetlík, R., Cech, J. \& Matas, J. Non-contact reflectance photoplethysmography: Progress, limitations, and myths. In 2018 13th IEEE International Conference on Automatic Face \& Gesture Recognition (FG 2018), 702-709 (IEEE, 2018).

66. Theofanopoulos, P. C. \& Trichopoulos, G. C. A novel fingerprint scanning method using terahertz imaging. In 2018 IEEE International Symposium on Antennas and Propagation \& USNC/URSI National Radio Science Meeting, 2463-2464 (IEEE, 2018).

67. Feldman, Y. et al. The electromagnetic response of human skin in the millimetre and submillimetre wave range. Phys. Medicine \& Biol. 54, 3341 (2009).

68. Zurich, E. IT IS Foundation Database (1996 (accessed August 31, 2014)).

69. Orfanidis, S. J. Electromagnetic waves and antennas. (2002).

\section{Acknowledgements}

Acknowledgements should be brief, and should not include thanks to anonymous referees and editors, or effusive comments. This work was supported in part by Center for Wireless Information Systems and Computational Architectures (WISCA), Arizona State University, Tempe.

\section{Author contributions statement}

Y.R., P.C.T, G.C.T. and D.W.B conceived the experiment(s), Y.R. and P.C.T conducted the experiments, Y.R. and P.C.T analysed the results. All authors reviewed the manuscript. 